\title{
Enhancing scalar productions with leptoquarks at the LHC
}

\author{
Arvind Bhaskar@, ${ }^{1, *}$ Debottam Das, ${ }^{2,3, \dagger}$ Bibhabasu De, ${ }^{2,3, *}$ and Subhadip Mitra $\oplus^{1, \S}$ \\ ${ }^{1}$ Center for Computational Natural Sciences and Bioinformatics, International Institute of Information \\ Technology, Hyderabad 500 032, India \\ ${ }^{2}$ Institute of Physics, Sachivalaya Marg, Bhubaneswar 751 005, India \\ ${ }^{3}$ Homi Bhabha National Institute, Training School Complex, Anushakti Nagar, Mumbai 400 085, India
}

(Received 2 May 2020; revised 15 July 2020; accepted 17 July 2020; published 4 August 2020)

The Standard Model (SM), when extended with a leptoquark (LQ) and right-handed neutrinos, can have interesting new implications for Higgs physics. We show that sterile neutrinos can induce a boost to the down-type quark Yukawa interactions through a diagonal coupling associated with the quarks and a scalar LQ of electromagnetic charge $1 / 3$. The relative change is moderately larger in the case of the first two generations of quarks, as they have vanishingly small Yukawa couplings in the SM. The enhancement in the couplings would also lead to a non-negligible contribution from the quark fusion process to the production of the $125 \mathrm{GeV}$ Higgs scalar in the SM, though the gluon fusion always dominates. However, this may not be true for a general scalar. As an example, we consider a scenario with a SM-gauge-singlet scalar $\phi$ where an $\mathcal{O}(1)$ coupling between $\phi$ and the LQ is allowed. The $\phi q \bar{q}$ Yukawa couplings can be generated radiatively only through a loop of LQ and sterile neutrinos. Here, the quark fusion process can have a significant cross section, especially for a light $\phi$. It can even supersede the normally dominant gluon fusion process for a moderate to large value of the LQ mass. This model can be tested/constrained at the high luminosity run of the LHC through a potentially large branching fraction of the scalar to two jets.

DOI: 10.1103/PhysRevD.102.035002

\section{INTRODUCTION}

The discovery of a Standard Model-(SM-)like Higgs boson of mass $125 \mathrm{GeV}$ at the LHC [1,2] and the subsequent measurements of its couplings to other SM particles have played a significant role in understanding the possible physics beyond the Standard Model (BSM). The Higgs couplings to the third generation fermions and the vector bosons have already been measured within $10 \%-20 \%$ of their SM predictions [3]. However, it is difficult to put strong bounds on the Yukawa couplings $\left(y_{f}\right)$ of the first two generations of fermions. This is an interesting point since, at the LHC, a change in the light-quark Yukawa couplings opens up the possibility of light quarks contributions to the

\footnotetext{
*arvind.bhaskar@research.iiit.ac.in

†debottam@iopb.res.in

tbibhabasu.d@iopb.res.in

§subhadip.mitra@iiit.ac.in
}

Published by the American Physical Society under the terms of the Creative Commons Attribution 4.0 International license. Further distribution of this work must maintain attribution to the author(s) and the published article's title, journal citation, and DOI. Funded by SCOAP. production of a Higgs. It motivates us to investigate whether it is possible to enhance the Yukawa couplings of the first two generation quarks in some existing minimal extension of the SM.

Therefore, in this paper, we study a simple extension of the SM augmented with a scalar leptoquark (LQ) of electromagnetic charge $1 / 3$ (generally denoted as $S_{1}$ ) and right-handed neutrinos. We find that the Yukawa couplings of the down-type quarks receive some new contributions and, for perturbative values of the free coupling parameters, can be moderately enhanced, especially for a SM-like Higgs $\left(h_{125}\right)$. However, for a singlet Higgs $(\phi)$, this enhancement could be more significant and could open up the $q \bar{q} \rightarrow \phi$ production channel. Here, we systematically study the production of both $h_{125}$ and $\phi$ at the $14 \mathrm{TeV}$ LHC through the quark and gluon fusion channels in the presence of a $S_{1}$ and right-handed neutrinos.

LQs are bosons that couple simultaneously to a quark and a lepton. They appear quite naturally in several extensions of the SM, especially in theories of grand unification like the Pati-Salam model [4], $S U(5)$ [5], or $S O(10)$ [6] (for a review, see [7]). Though, in principle, LQs can be either scalar or vector in local quantum field theories, the scalar states are more attractive, as the vector 
ones may lead to some problems with loops [8-10]. In recent times, LQ models (with or without right-handed neutrinos) have drawn attention for various reasons. For example, they can be used to explain different B-meson anomalies [11-20] or to enhance flavor violating decays of Higgs and leptons like $\tau \rightarrow \mu \gamma$ and $h \rightarrow \tau \mu$ [21]. LQs may also play a role to accommodate dark matter abundance $[22,23]$ or to mitigate the discrepancy in the anomalous magnetic moment of muon $(g-2)_{\mu}$ [24-26]. Direct production of $\mathrm{TeV}$ scale right-handed neutrinos at the LHC can be strongly enhanced if one considers the neutrino mass generated at tree level via the inverse-seesaw mechanism within LQ scenarios [27]. An $S_{1}$-Higgs coupling can help to stabilize the electroweak vacuum [28]. The collider phenomenology of various LQs has also been extensively discussed in the literature [7,29-38].

In the scenario that we consider, there are three generations of right chiral neutrinos in addition to the $S_{1}$. Generically, such a scenario is not very difficult to realize within the grand unified frameworks. In fact, considering sterile neutrinos in this context is rather well motivated because of the existence of nonzero neutrino masses and mixings, which have been firmly established by now. It is known that an $\mathcal{O}(1)$ Yukawa coupling between the chiral neutrinos and $\mathrm{TeV}$ scale masses for the right-handed neutrinos can explain the experimental observations related to neutrino masses and mixing angles even at tree level if one extends SM to a simple setup like the inverse seesaw mechanism (ISSM) [39-41]. Of course, this requires the presence of an additional singlet neutrino state $X$ in the model. ${ }^{1}$

Interestingly, the production cross sections of sterile neutrinos at the LHC can be enhanced significantly if the ISSM is embedded in a LQ scenario [27]. Similarly, as indicated earlier, a $\nu_{R}$ state in a loop accompanied with $S_{1}$ may influence the production of a Higgs at the LHC and its decays to the SM fermions, especially to the light ones. Observable effects can be seen in scenarios with a general scalar sector that may include additional Higgs states, a TeV scale $\nu_{R}$, and an $\mathcal{O}(1)$ Yukawa couplings between the left and right chiral neutrinos. In this paper, we shall explore this in some detail. Notably, the gluon fusion process $(\mathrm{ggF})$ for producing a Higgs scalar gets boosted in presence of a LQ [51]. Our study is generalit can be applied to both SM-like and BSM Higgs bosons. Specifically, we consider the following two cases:

(a) A $125 \mathrm{GeV}$ SM-like Higgs boson $\left(h_{125}\right)$. We investigate how the light-quark Yukawa couplings can get some positive boosts. However, obtaining a free rise of the Yukawa parameters is not possible in

${ }^{1}$ ISSM or inverse seesaw extended supersymmetric models may lead to interesting phenomenology at low energy [42-50]. our model ${ }^{2}$ and, as we shall see, for perturbative new couplings and $\mathrm{TeV}$ scale new physics masses, the boosts are moderate and lead to some enhancement of both production and decays of $h_{125}$ at the LHC.

(b) A singlet scalar $\phi$ (BSM Higgs). We also study the productions and decays of a scalar $\phi$ that is a singlet under the SM gauge group. Such a scalar has been considered in different contexts in the literature. For example, it may serve as a dark matter candidate. Similarly, a singlet scalar can help solve the so-called $\mu$ problem in the Minimal Supersymmetric Standard Model [55]. To produce such a singlet at the LHC, one generally relies upon its mixing with the doubletlike Higgs states present in the theory. If the mixing is nonnegligible, then the leading order production process turns out to be the gluon fusion (though vector boson fusion may also become relevant in specific cases [56]). One may also consider the production of $\phi$ through cascade decays of the doublet Higgs state(s). However, such a process is generally much suppressed. Now, as we shall see, in the presence of a scalar LQ and sterile neutrinos we could have a new loop contribution to the quark fusion production process (qqF). The LQ would also contribute to the gluon fusion process. In such a setup, the singlet Higgs can potentially be tested at the LHC via its decays to the light-quark states.

The rest of the paper is organized as follows. In Sec. II, we introduce the model Lagrangian and discuss the new interactions. In Sec. III, we discuss additional contributions

\footnotetext{
${ }^{2}$ This may be possible in an effective theory with free parameters. For example, Ref. [52] considers a dimension-6 operator of the form $f_{d}\left(H^{\dagger} H / \Lambda^{2}\right)\left(\bar{q}_{L} H d_{R}\right)+$ H.c. (where $\Lambda \sim \mathrm{TeV})$ in addition to the SM Yukawa terms that contribute differently to the physical quark masses and effective quark Yukawa couplings. Thus, by choosing $f_{d}$ one may raise the Yukawa parameters while keeping the physical masses unchanged, though this may require some fine-tuning among the parameters of the model. It is important to note that in the presence of higher-dimensional operators, a large Yukawa coupling need not induce large correction to the corresponding quark mass always.

Such enhancements of the light-quark Yukawa couplings can even be probed at the LHC. An analysis of Higgs boson pair production suggests that in the future the High Luminosity LHC (HL-LHC) may offer a handle on this [53]. An updated analysis, with $3000 \mathrm{fb}^{-1}$ of integrated luminosity suggests (though not in a fully model independent way) that it may be possible to narrow down the $d$-and $s$-quark Yukawa couplings to about 260 and 13 times to their SM values, respectively [54], i.e.,
}

$$
\left|\kappa_{d}\right| \leq 260, \quad\left|\kappa_{s}\right| \leq 13
$$

where the Yukawa coupling modifier $\kappa_{q}$ is defined as

$$
\kappa_{q}=\frac{y_{q}^{\mathrm{eff}}}{y_{q}^{\mathrm{SM}}} .
$$


to the production and decays of $h_{125}$. In Sec. IV, we discuss bounds on the parameters. In Sec. V, we investigate the case of the singlet scalar $\phi$. Finally we summarize our results and conclude in Sec. VI.

\section{THE MODEL: A SIMPLE EXTENSION OF THE SM}

As explained in the Introduction, our model is a simple extension of the SM with chiral neutrinos and an additional scalar LQ of electromagnetic charge 1/3, normally denoted as $S_{1}$. The LQ transforms under the SM gauge group as $(\overline{\mathbf{3}}, \mathbf{1}, 1 / 3)$ with $Q_{\mathrm{EM}}=T_{3}+Y$. In the notation of Ref. [7], the general fermionic interaction Lagrangian for $S_{1}$ can be written as

$$
\begin{aligned}
\mathcal{L}_{F}= & \left(y_{1}^{L L}\right)_{i j}\left(\bar{Q}_{L}^{C i a} \epsilon^{a b} L_{L}^{j b}\right) S_{1}+\left(y_{1}^{R R}\right)_{i j}\left(\bar{u}_{R}^{C i} e_{R}^{j}\right) S_{1} \\
& +\left(y_{1}^{\overline{R R}}\right)_{i j}\left(\bar{d}_{R}^{C i} \nu_{R}^{j}\right) S_{1}+\text { H.c. },
\end{aligned}
$$

where we have suppressed the color indices. The superscript $C$ denotes charge conjugation; $\{i, j\}$ and $\{a, b\}$ are flavor and $S U(2)$ indices, respectively. The SM quark and lepton doublets are denoted by $Q_{L}$ and $L_{L}$, respectively. We now add the scalar interaction terms to the Lagrangian in Eq. (3),

$$
\begin{aligned}
\mathcal{L} \supset & \mathcal{L}_{F}+\lambda\left(H^{\dagger} H\right)\left(S_{1}^{\dagger} S_{1}\right)+\lambda^{\prime} \phi\left(S_{1}^{\dagger} S_{1}\right) \\
& +\mu\left(H^{\dagger} H\right) \phi^{2}+\frac{1}{2} M_{\phi}^{2} \phi^{2}+\bar{M}_{S_{1}}^{2}\left(S_{1}^{\dagger} S_{1}\right) .
\end{aligned}
$$

Here, $H$ denotes the SM Higgs doublet, and $M_{\phi}$ and $\bar{M}_{S_{1}}$ define the bare mass parameters for $\phi$ and $S_{1}$, respectively. We denote the physical Higgs field after the electroweak symmetry breaking as $h \equiv h_{125}$. The singlet $\phi$ does not acquire any vacuum expectation value (VEV). Physical masses can be obtained via

$$
H=\frac{1}{\sqrt{2}}\left(\begin{array}{c}
0 \\
v+h
\end{array}\right), \quad \phi=\phi,
$$

where the SM Higgs VEV $v \simeq 246 \mathrm{GeV}$. We assume the mixing between $H$ and $\phi$, controlled by the dimensionless coupling $\mu$ to be small to ensure that the presence of a singlet Higgs does not affect the production and decays of $h_{125}$ significantly via mixing. Notice that, unlike dimensionless $\lambda$ or $\mu, \lambda^{\prime}$ is a dimension-1 parameter. We define the physical mass of $S_{1}$ to be $M_{S_{1}}$ as

$$
M_{S_{1}}^{2}=\bar{M}_{S_{1}}^{2}+\frac{1}{2} \lambda v^{2}
$$

The above Lagrangian simplifies a bit if we ignore the mixing among quarks and neutrinos (i.e., set $\mathbf{V}_{\mathrm{CKM}}=$ $\mathbf{U}_{\mathrm{PMNS}}=\mathbb{I}$ ). For example, we can expand Eq. (4) for the first generation as

$$
\begin{aligned}
\mathcal{L} \supset & \left\{y_{1}^{L L}\left(-\bar{d}_{L}^{C} \nu_{L}+\bar{u}_{L}^{C} e_{L}\right) S_{1}+y_{1}^{R R} \bar{u}_{R}^{C} e_{R} S_{1}\right. \\
& \left.+y_{1}^{\overline{R R}} \bar{d}_{R}^{C} \nu_{R} S_{1}+\text { H.c. }\right\}+\lambda v h\left(S_{1}^{\dagger} S_{1}\right) \\
& +\lambda^{\prime} \phi\left(S_{1}^{\dagger} S_{1}\right)+\frac{1}{2} M_{\phi}^{2} \phi^{2}+M_{S_{1}}^{2}\left(S_{1}^{\dagger} S_{1}\right),
\end{aligned}
$$

where we have simplified $\left(y_{1}^{X}\right)_{i i}$ as $y_{i}^{X}$. Since the flavor of the neutrino is irrelevant for the LHC, from here on we shall simply write $\nu$ to denote neutrinos.

The terms in Eq. (7) have the potential to boost up some production/decay modes for $h$ and $\phi$. For example, it would lead to an additional contribution to the effective $h g g$ coupling (see Fig. 1) [51]. Similarly, the decay $h \rightarrow d \bar{d}$, which is negligible in the SM, may get a boost now, as long as some of the new couplings are not negligible. The processes are illustrated in Figs. 1(a)-1(c) [the first diagram is independent of $v$, while the other two are of $\mathcal{O}\left(v^{2}\right)$ ], where the Higgs is shown to be decaying to a $d \bar{d}$ pair via a triangle loop mediated by $S_{1}$ and chiral neutrinos. There are two possibilities: the Higgs directly couples with either the chiral neutrinos or the LQ. Since the contributions of these diagrams appear as corrections to $y_{d}$, it is easy to see that the fermion in the loop (i.e., the neutrino) has to go through a chirality flip. In this case, the right-handed neutrino from the third term in Eq. (3) helps to get a nonzero contribution.

One can, of course, imagine similar diagrams with charged leptons in the loops, contributing to the $h \rightarrow u \bar{u}$ (or any other up-type quark-antiquark pair) decay. However, the contributions of such diagrams would be small as they are suppressed by the tiny charged lepton Yukawa couplings, at least for the first two generations. If we restrict ourselves only to flavor diagonal couplings in Eq. (3) (i.e., we allow only $i=j$ terms), only the top Yukawa $y_{t}$ would be modified appreciably. If we allow offdiagonal couplings, one can get contributions for the first two generations of Yukawa couplings-namely, $y_{u}$ and $y_{c}$, respectively. However, one needs to be careful as offdiagonal LQ-quark-lepton couplings are constrained, particularly for the first two generations [7,57]. In this case, we consider only flavor diagonal couplings and look only at the modification of Higgs couplings to down-type quarks. Thus, one may always set $\left(y_{1}^{R R}\right)_{i j}=0$ for all values of $i$ and $j$. This may lead to a somewhat favorable situation in some cases to accommodate rare decays of fermions through LQ exchange.

Before we discuss productions and decays of $h_{125}$ and $\phi$ in our model, a few comments are in order. As we shall see in the next section, an order $1 h \bar{\nu}_{L} \nu_{R}$ coupling, i.e., $y_{\nu} \sim$ $\mathcal{O}(1)$ and a TeV scale mass for the $\nu_{R}$ would be helpful to raise the Yukawa couplings of the light quarks. Typically, the models like ISSM would be able to accommodate such a scenario. In the ISSM, an additional gauge singlet neutrino, usually denoted by $X$, is assigned a Majorana mass term $\mu_{X} X X$, while $\nu_{R}$ receives a Dirac mass term of 
the form $M \nu_{R}^{-} X$. For our purposes, we may assume that this singlet $X$ cannot directly interact with any other particle we consider. However, since it interacts exclusively with the $\nu_{R}$ fields via $M$, it would modify the $\nu_{R}$ propagators. In this case, it may be useful to define something called a "fat $\nu_{R}$ propagator" [58] that includes all the effects of the sequential insertions of the $X$ field. For simplicity, we do not display this interaction and mass term of the righthanded neutrinos explicitly in Eq. (3). One can explicitly consider an ISSM in the backdrop of our analysis and easily accommodate fat $\nu_{R}$ propagators without any change in our results.

\section{CONTRIBUTION TO THE PRODUCTION AND DECAYS OF $\boldsymbol{h}_{125}$}

In this section, we first look into the additional contributions to the Yukawa couplings of the down-type quarks with $h_{125}$. The relevant interactions can be read from Eq. (7). We shall then discuss the role of these loops in the production of $h_{125}$ and its decays to the down-type quarks. In this paper, we compute all the loop diagrams using dimensional regularization and Feynman parametrization and then match the results using the PassarinoVeltman (PV) integrals [59]. We evaluate the PV integrals with two publicly available packages, FeynCalc [60] and LoopTools [61].

\section{A. Correction to Yukawa couplings of the down-type quarks}

In our calculation, we assume that left-handed neutrinos are massless while right-handed ones are massive. Also, since we consider Higgs decays to down-type quarks only, we can safely ignore the quark masses $\left(m_{q}=0\right)$ and set $m_{h}^{2}=\left(p_{1}+p_{2}\right)^{2}=2 p_{1} \cdot p_{2}$ (see Fig. 1). The correction to $y_{d}$ coming from the diagram shown in Fig. 1(a) is given by

$$
\begin{aligned}
\tilde{y}_{d}^{(a)}= & -i g_{1}^{2} y_{\nu} \int \frac{d^{4} \ell}{(2 \pi)^{4}}\left[\frac{P_{R} \mathscr{\ell}\left(\not p_{1}+\not \not_{2}-\not \ell+M_{\nu_{R}}\right) P_{R}}{\ell^{2}\left\{\left(p_{1}+p_{2}-\ell\right)^{2}-M_{\nu_{R}}^{2}\right\}}\right. \\
& \left.\times \frac{1}{\left\{\left(p_{1}-\ell\right)^{2}-M_{S_{1}}^{2}\right\}}\right],
\end{aligned}
$$

where $g_{1}^{2}=g_{L} g_{R}=y_{1}^{L L} y_{1}^{R \bar{R}}$ and $P_{L / R}$ are the chirality projectors. From here on, we shall suppress the generation index of the leptoquark couplings and simply write $g_{i}^{2}$ as $g^{2}$. After Feynman parametrization and dimensional regularization, we get

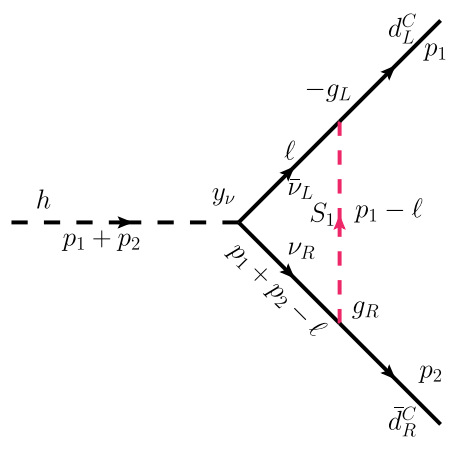

(a)

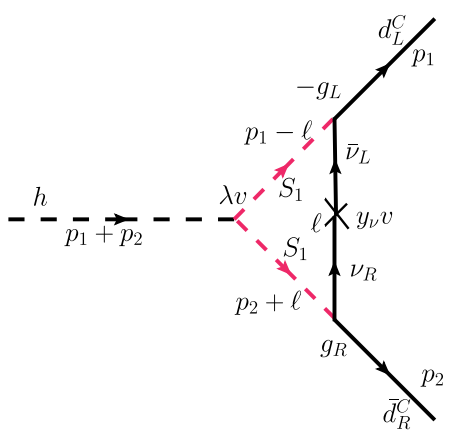

(b)

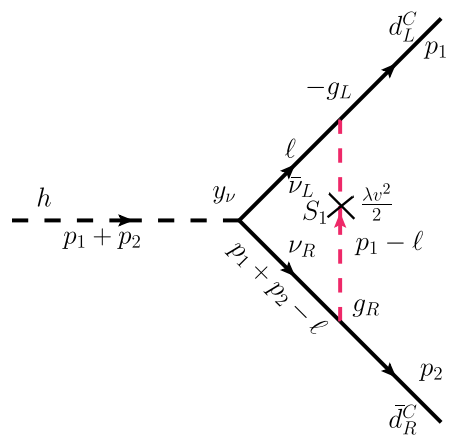

(c)

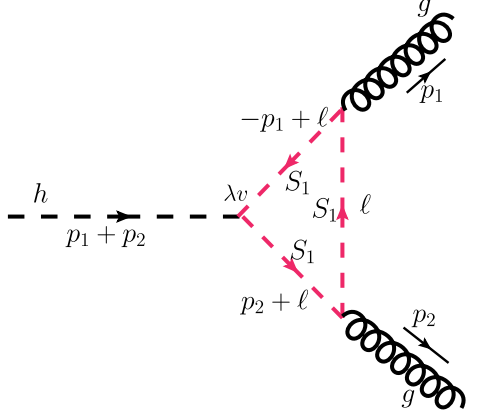

(d)

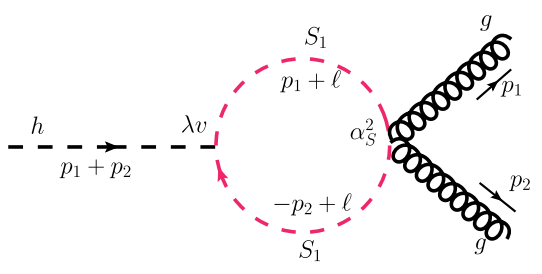

(e)

FIG. 1. Feynman diagrams showing the SM-like Higgs $\left(h_{125}\right)$ decaying to (a)-(c) down quarks and (d), (e) gluon pairs through loop diagrams mediated by $S_{1}$ and chiral neutrinos. Only in (a) and (c) does the Higgs couple to $\nu$, whereas it couples to $S_{1}$ in all the other diagrams. The couplings $g_{L}=y_{1}^{L L}$ and $g_{R}=y_{1}^{\overline{R R}}$ [see Eq. (7)]. The diagrams for $s$ - and $b$-quarks are similar to the last two diagrams. Note that we absorb a factor of $1 / \sqrt{2}$ in the definition of Yukawa couplings in the mass basis, i.e., we write $y_{\nu}$ instead of $y_{\nu} / \sqrt{2}$. 


$$
\begin{aligned}
\tilde{y}_{d}^{(a)}= & -\frac{g^{2} y_{\nu}}{16 \pi^{2}}\left[\int_{0}^{1} d x \int_{0}^{1-x} d y\left(\frac{x m_{h}^{2}}{D_{1}}\right)\right. \\
& \left.-\int_{0}^{1} d z \ln D_{2}+\Delta_{\epsilon}\right],
\end{aligned}
$$

where

$$
D_{1}(x, y)=x y m_{h}^{2}+x(x-1) m_{h}^{2}+x M_{\nu_{R}}^{2}+y M_{S_{1}}^{2}
$$

and

$$
D_{2}(z)=z M_{S_{1}}^{2}+(1-z) M_{\nu_{R}}^{2}
$$

The divergent piece at $\mathcal{O}\left(v^{0}\right), \Delta_{\epsilon}=\frac{2}{\epsilon}-\gamma+\ln (4 \pi)+\mathcal{O}(\epsilon)$ is canceled by a similar contribution from diagrams with a bubble in an external quark line. The bubble in the quark lines is obtained by replacing the Higgs field in Fig. 1(a) with $v$ and amputating the external quark lines; see Fig. 2 (a). This extra contribution is given as

$$
\left.y_{d}^{\operatorname{leg}}\right|_{\mathcal{O}\left(v^{0}\right)}=\frac{g^{2} y_{\nu}}{16 \pi^{2}} \int_{0}^{1} d z\left[\Delta_{\epsilon}-\ln \left\{z M_{S_{1}}^{2}+(1-z) M_{\nu_{R}}^{2}\right\}\right] .
$$

Putting these two together, we get

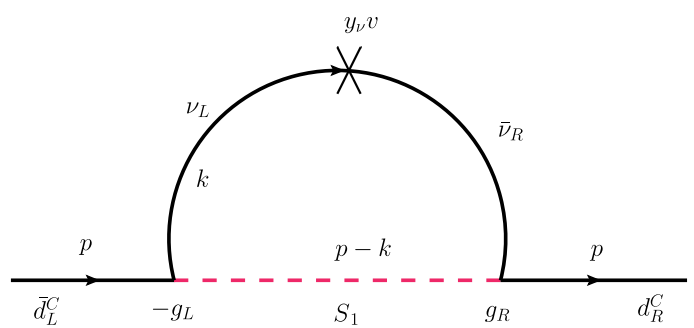

(a)

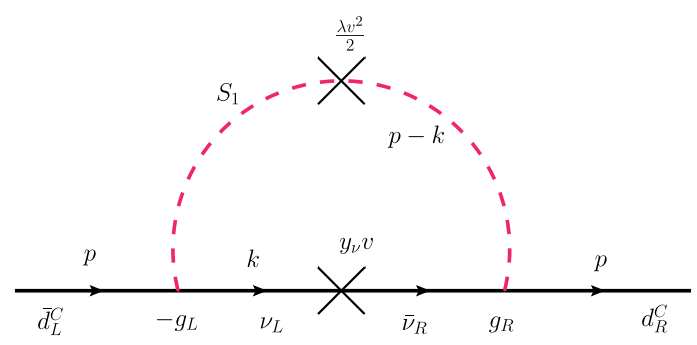

(b)

FIG. 2. Feynman diagrams showing the (a) $O\left(v^{0}\right)$ and (b) $O\left(v^{2}\right)$ corrections to the quark propagator from loop diagrams mediated by $S_{1}$ and chiral neutrinos. The couplings $g_{L}=y_{1}^{L L}$ and $g_{R}=y_{1}^{R \bar{R}}$ [see Eq. (7)]. The diagrams for $s$ - and $b$-quarks are similar. These corrections are independent of the external momentum $(p)$ and hence contribute as mass corrections.

$$
y_{d}^{(a)}=-\frac{g^{2} y_{\nu}}{16 \pi^{2}}\left[\int_{0}^{1} d x \int_{0}^{1-x} d y\left(\frac{x m_{h}^{2}}{D_{1}}\right)\right] .
$$

Now, proceeding along the same lines, we get the correction from the diagram in Fig. 1(b) as

$$
\begin{aligned}
y_{d}^{(b)}= & i g^{2} \lambda y_{\nu} v^{2} \int \frac{d^{4} \ell}{(2 \pi)^{4}}\left[\frac{1}{\left(\ell^{2}-M_{\nu_{R}}^{2}\right)\left\{\left(\ell-p_{1}\right)^{2}-M_{S_{1}}^{2}\right\}}\right. \\
& \left.\times \frac{1}{\left\{\left(\ell+p_{2}\right)^{2}-M_{S_{1}}^{2}\right\}}\right] \\
= & \frac{g^{2} \lambda y_{\nu} v^{2}}{16 \pi^{2}} \int_{0}^{1} d x \int_{0}^{1-x} d y\left(\frac{1}{D_{0}}\right)
\end{aligned}
$$

where $D_{0}(x, y)=M_{\nu_{R}}^{2}+(x+y)\left(M_{S_{1}}^{2}-M_{\nu_{R}}^{2}\right)-x y m_{h}^{2}$. Similarly, the correction term corresponding to Fig. 1(c) can be obtained as

$$
\begin{aligned}
\tilde{y}_{d}^{(c)}= & \frac{i g^{2} y_{\nu} \lambda v^{2}}{2} \int \frac{d^{4} \ell}{(2 \pi)^{4}}\left[\frac{\left.P_{R} \not \ell(\not)_{1}+\not p_{2}-\not \ell+M_{\nu_{R}}\right) P_{R}}{\ell^{2}\left\{\left(p_{1}+p_{2}-\ell\right)^{2}-M_{\nu_{R}}^{2}\right\}}\right. \\
& \left.\times \frac{1}{\left\{\left(p_{1}-\ell\right)^{2}-M_{S_{1}}^{2}\right\}^{2}}\right] \\
= & -\frac{g^{2} y_{\nu} \lambda v^{2}}{32 \pi^{2}}\left[\int_{0}^{1} d x \int_{0}^{1-x} d y(1-x-y)\left(\frac{y m_{h}^{2}}{D_{3}^{2}}\right)\right. \\
& \left.+\int_{0}^{1} d x \int_{0}^{1-x} d y\left(\frac{1}{D_{4}}\right)\right],
\end{aligned}
$$

where $D_{3}(x, y)=-x y m_{h}^{2}+y M_{\nu_{R}}^{2}+(1-x-y) M_{S_{1}}^{2}$ and $D_{4}(x)=x M_{\nu_{R}}^{2}+(1-x) M_{S_{1}}^{2}$. This is finite like $y^{(b)}$. The last term of Eq. (15) is actually canceled by the $\mathcal{O}\left(v^{2}\right)$ correction to the external quark propagators, as shown in Fig. 2(b). This is similar to the cancellation at $\mathcal{O}\left(v^{0}\right)$ in $y^{(a)}$; in this case, the bubble is obtained by replacing the Higgs field in Fig. 1(b) with $v$ and amputating the external quark lines. However, one has to be careful with the factors here. After electroweak symmetry breaking, one can expand the Higgs- $S_{1}$ interaction term in Eq. (4) as

$$
\lambda\left(H^{\dagger} H\right)\left(S_{1}^{\dagger} S_{1}\right)=\frac{\lambda}{2}\left(h^{2}+2 h v+v^{2}\right)\left(S_{1}^{\dagger} S_{1}\right)+\cdots .
$$

The $\lambda v h\left(S_{1}^{\dagger} S_{1}\right)$ term contributes to $y_{q}^{(b)}$, but the propagator correction would come from the $\lambda v^{2}\left(S_{1}^{\dagger} S_{1}\right) / 2$ term, i.e., with a different prefactor. The $\mathcal{O}\left(v^{2}\right)$ external leg correction to the Yukawa coupling is proportional to $\lambda v^{2}\left(S_{1}^{\dagger} S_{1}\right) / 2$ and can be written as

$$
\left.y_{d}^{\operatorname{leg}}\right|_{\mathcal{O}\left(v^{2}\right)}=\frac{g^{2} y_{\nu} \lambda v^{2}}{32 \pi^{2}} \int_{0}^{1} d x\left(\frac{1-x}{x M_{\nu_{R}}^{2}+(1-x) M_{S_{1}}^{2}}\right) .
$$

Once this is added, we get 
$y_{d}^{(c)}=-\frac{g^{2} y_{\nu} \lambda v^{2}}{32 \pi^{2}} \int_{0}^{1} d x \int_{0}^{1-x} d y(1-x-y)\left(\frac{y m_{h}^{2}}{D_{3}^{2}}\right)$.

Therefore, the effective $h d \bar{d}$ coupling can be written as

$$
\begin{aligned}
y_{d}^{\mathrm{eff}}= & y_{d}^{\mathrm{SM}}+\delta y \\
= & y_{d}^{\mathrm{SM}}+\frac{g^{2} y_{\nu}}{16 \pi^{2}}\left[\int _ { 0 } ^ { 1 } d x \int _ { 0 } ^ { 1 - x } d y \left\{\frac{\lambda v^{2}}{D_{0}}-\frac{x m_{h}^{2}}{D_{1}}\right.\right. \\
& \left.\left.-(1-x-y)\left(\frac{\lambda v^{2} \times y m_{h}^{2}}{2 D_{3}^{2}}\right)\right\}\right],
\end{aligned}
$$

where $y_{d}^{\mathrm{SM}}=m_{d} / v$ is the $d$-quark Yukawa coupling in the $\mathrm{SM}$ (with $m_{d}$ being the physical mass) and $\delta y=y_{d}^{(a)}+$ $y_{d}^{(b)}+y_{d}^{(c)}$ is the total loop correction. This results in a finite shift to the SM down-quark Yukawa couplings which cannot be absorbed in a redefinition of the quark masses since the corrections corresponding to the mass terms (Fig. 2) are already accounted for in $m_{d}$, the physical mass, through Eqs. (12) and (17). 'This is similar to the case in which the SM is augmented with dimension-6 operators [52].

Equation (19) can also be written in terms of the following PV integrals,

$$
\begin{aligned}
y_{d}^{\mathrm{eff}}= & y_{d}^{\mathrm{SM}}+\frac{g^{2} y_{\nu}}{16 \pi^{2}}\left[B_{0}\left(0, M_{\nu_{R}}^{2}, M_{S_{1}}^{2}\right)-B_{0}\left(m_{h}^{2}, 0, M_{\nu_{R}}^{2}\right)\right. \\
& -M_{S_{1}}^{2} C_{0}\left(0,0, m_{h}^{2}, 0, M_{S_{1}}^{2}, M_{\nu_{R}}^{2}\right) \\
& -\lambda v^{2} C_{0}\left(0,0, m_{h}^{2}, M_{S_{1}}^{2}, M_{\nu_{R}}^{2}, M_{S_{1}}^{2}\right) \\
& +\frac{\lambda v^{2}}{2}\left\{C_{0}\left(0,0, m_{h}^{2}, 0, M_{S_{1}}^{2}, M_{\nu_{R}}^{2}\right)\right. \\
& +M_{S_{1}}^{2} D_{0}\left(0,0, m_{h}^{2}, 0,0,0, M_{S_{1}}^{2}, M_{S_{1}}^{2}, 0, M_{\nu_{R}}^{2}\right) \\
& \left.\left.-C_{0}\left(0,0,0, M_{S_{1}}^{2}, M_{S_{1}}^{2}, M_{\nu_{R}}^{2}\right)\right\}\right],
\end{aligned}
$$

where $D_{0}, C_{0}$, and $B_{0}$ are the four-point, triangle, and bubble integrals, respectively. The expressions for the $s$ and $b$-quarks would be exactly the same as the above with $m_{d}$ and $g^{2}=g_{i}^{2}$ suitably modified.

\section{B. Relative couplings}

To get some idea about how the extra contributions from the loops depend on the parameters, we use the Yukawa coupling modifiers [Eq. (2)],

$$
\kappa_{q}=1+\frac{\delta y}{y_{q}^{\mathrm{SM}}} .
$$

Since we ignore the mass of the quarks, $\delta y$ is independent of the flavor of the down-type quark that the Higgs is coupling to as long as $g^{2} y_{\nu}$ remains the same. Hence, $\delta y / y_{q}^{\mathrm{SM}}$ should go as $1 / y_{q}^{\mathrm{SM}} \sim 1 / m_{q}$. Using this and
TABLE I. Contributions of the three diagrams shown in Fig. 1 to the Yukawa couplings obtained from Eq. (19) or (20) for some illustrative choices of the mass of the right-handed neutrino $M_{\nu_{R}}$ and the leptoquark mass $M_{S_{1}}$ while keeping $g^{2} y_{\nu}=1$ and $\lambda=1$.

\begin{tabular}{lcccc}
\hline \hline$M_{\nu_{R}}(\mathrm{GeV})$ & $M_{S_{1}}(\mathrm{GeV})$ & $y^{(a)}$ & $y^{(b)}$ & $y^{(c)}$ \\
\hline 600 & 1000 & -0.000046 & 0.000255 & $-6.3 \times 10^{-7}$ \\
& 1500 & -0.000031 & 0.000132 & $-2.2 \times 10^{-7}$ \\
1100 & 1000 & -0.000022 & 0.000180 & $-2.1 \times 10^{-7}$ \\
& 1500 & -0.000016 & 0.000103 & $-0.9 \times 10^{-7}$ \\
\hline \hline
\end{tabular}

Eq. (20), we see that $\kappa_{q}$ depends linearly on $1 / m_{q}, \lambda$, and the combination $g^{2} y_{\nu}$, but, a priori, its dependence on $M_{S_{1}}$ or $M_{\nu_{R}}$ is not so simple. In Table I, we show the contributions of the three loop diagrams [Figs. 1(a)-1(c)] for some illustrative choices of $M_{\nu_{R}}$ and $M_{S_{1}}$. With $g^{2} y_{\nu}=\lambda=1$, we see that there is some cancellation between these contributions. Note that this choice of coupling is not restricted by the rare decays $[7,57]$.

In Fig. 3, we show the variations of $\kappa_{d}, \kappa_{s}$, and $\kappa_{b}$ for $500 \leq M_{S_{1}} \leq 3000 \mathrm{GeV}$ for three different choices of $M_{\nu_{R}}$. As expected, we see the lightest among the three quarks, i.e., the $d$-quark, getting the maximum deviation in $\kappa_{q}$ from unity. The $b$-quark coupling hardly moves from the SM value for the considered parameter range. However, all the deviations are well within the ranges allowed by Eq. (1).

\section{Decays of $\boldsymbol{h}_{\mathbf{1 2 5}}$}

As mentioned before, we shall use $h$ and $h_{125}$ interchangeably to denote the $125 \mathrm{GeV}$ SM-like Higgs boson. In the SM, the total decay width of the $125 \mathrm{GeV}$ Higgs boson is computed as $\Gamma_{h}^{\mathrm{SM}}=4.07 \times 10^{-3} \mathrm{GeV}$, with a relative theoretical uncertainty of ${ }_{-3.9 \%}^{+4.0 \%}$ [62]. Now, because of the additional loop contribution, the total decay width would increase in our model. We can use Eq. (19) or (20) to compute the partial decay width for the $h \rightarrow q \bar{q}$ decay in the rest frame of the Higgs as

$$
\begin{aligned}
\Gamma_{h \rightarrow q \bar{q}} & =N_{c} \times \frac{\left|\overrightarrow{p_{q}}\right|}{32 \pi^{2} m_{h}^{2}} \int\left|\mathcal{M}_{\mathrm{tot}}\right|^{2} d \Omega \\
& =\frac{N_{c}}{8 \pi m_{h}^{2}}\left|y_{q}^{\mathrm{eff}}\right|^{2}\left(m_{h}^{2}-4 m_{q}^{2}\right)^{3 / 2},
\end{aligned}
$$

where $i \mathcal{M}_{\text {tot }}=y_{q}^{\text {eff }} q \bar{q}$ is the invariant amplitude and $N_{c}=$ 3 accounts for the colors of the quark. Similarly, the $h \rightarrow g g$ partial width would also get a positive boost in the presence of $S_{1}$ [7]. The relevant diagrams can be seen in Figs. 1(d) and 1(e). In our model, the $h \rightarrow g g$ partial width can be expressed as $[7,63]$,

$$
\Gamma_{h \rightarrow g g}=\frac{G_{\mathrm{F}} \alpha_{s}^{2} m_{h}^{3}}{64 \sqrt{2} \pi^{3}}\left|\mathcal{A}_{1 / 2}\left(x_{t}\right)+\frac{\lambda v^{2}}{2 M_{S_{1}}^{2}} \mathcal{A}_{0}\left(x_{S_{1}}\right)\right|^{2},
$$




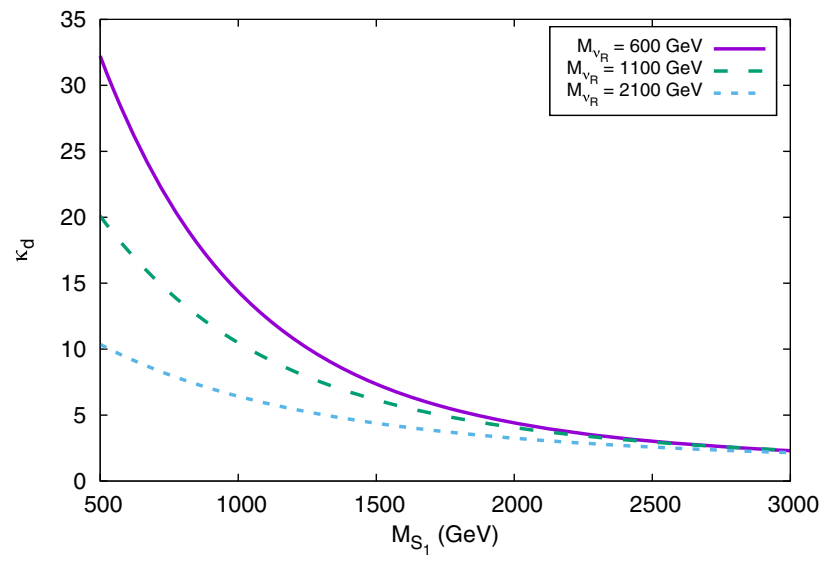

(a)

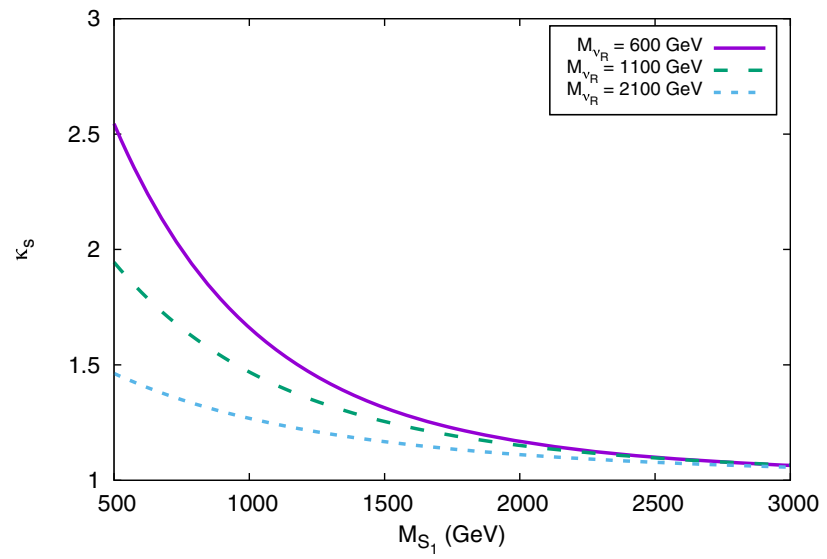

(b)

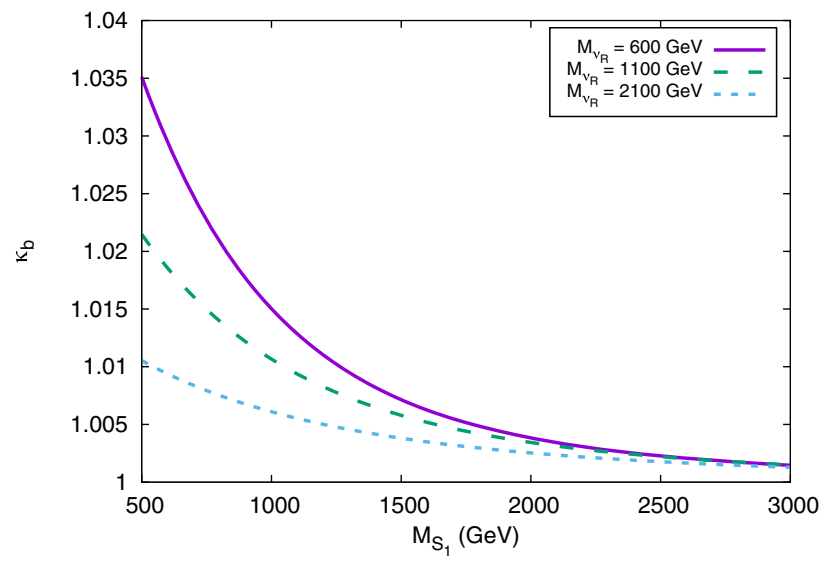

(c)

FIG. 3. Variation of the coupling modifiers (a) $\kappa_{d}$, (b) $\kappa_{s}$, and (c) $\kappa_{b}$ [defined in Eq. (2)] with $M_{S_{1}}$ for different values of $M_{\nu_{R}}$. Here we set $g^{2} y_{\nu}=1$ for all three generations and keep $\lambda=1$.

where $x_{t}=m_{h}^{2} / 4 m_{t}^{2}$ and $x_{S_{1}}=m_{h}^{2} / 4 M_{S_{1}}^{2}$. The relevant one-loop functions are given by

$$
\mathcal{A}_{1 / 2}(x)=\frac{2[x+(x-1) f(x)]}{x^{2}},
$$

$$
\begin{gathered}
\mathcal{A}_{0}(x)=-\frac{[x-f(x)]}{x^{2}}, \\
f(x)=\left\{\begin{array}{ll}
\arcsin ^{2}(\sqrt{x}), & x \leq 1 \\
-\frac{1}{4}\left[\ln \left(\frac{1+\sqrt{1-x^{-1}}}{1-\sqrt{1-x^{-1}}}\right)-i \pi\right]^{2}, & x>1
\end{array}\right\} .
\end{gathered}
$$

Now, Eqs. (22) and (23) can be used to obtain the total width in our model,

$$
\begin{aligned}
\Gamma_{h}= & \left(\Gamma_{h}^{\mathrm{SM}}-\Gamma_{h \rightarrow g g}^{\mathrm{SM}}-\sum_{q=d, s, b} \Gamma_{h \rightarrow q \bar{q}}^{\mathrm{SM}(\mathrm{tree})}\right)+\Gamma_{h \rightarrow g g} \\
& +\sum_{q=d, s, b} \Gamma_{h \rightarrow q \bar{q}}
\end{aligned}
$$

Ideally, we should also include corrections to partial widths of other decay modes, like $h \rightarrow \gamma \gamma$ or other three body decays in the above expression. However, since their contributions to the total width are relatively small, we ignore them.

From Eqs. (22) and (27), we compute the new branching ratios (BRs) of the $h \rightarrow q \bar{q}$ modes in our model as

$$
\operatorname{BR}(h \rightarrow q \bar{q})=\frac{\Gamma_{h \rightarrow q \bar{q}}}{\Gamma_{h}} .
$$

In Fig. 4, we show $\operatorname{BR}(h \rightarrow q \bar{q})$ for different quarks for $g^{2} y_{\nu}=1$ (for all generations) and $\lambda=1$. Equation (22) indicates $\operatorname{BR}(h \rightarrow q \bar{q}) \sim\left|y_{q}^{\mathrm{SM}}+\delta y\right|^{2}$; i.e., it increases with $y_{q}^{\mathrm{SM}}$ (remember, for $g^{2} y_{\nu}=1, \delta y$ is the same for all the quarks). Hence, we expect $\operatorname{BR}(h \rightarrow b \bar{b})>$ $\mathrm{BR}(h \rightarrow s \bar{s})>\mathrm{BR}(h \rightarrow d \bar{d})$, as $y_{q}^{\mathrm{SM}}$ increases with the mass of the quark. This can be seen in Fig. 4. However, even for order $1 y_{\nu}$ couplings and TeV scale $S_{1}$ and $\nu_{R}$, the relative shift in branching ratio of the $h \rightarrow b \bar{b}$ decay to that of SM is not large (as expected from Fig. 3). For the lighter quarks, the branching ratios become much larger than their SM values, even though they remain small compared to other decay modes like $h \rightarrow b \bar{b}$. The branching fraction $h \rightarrow g g$ is almost unaffected with the variation in $S_{1}$, as the SM contribution always dominates.

\section{Production of $\boldsymbol{h}_{125}$}

For a quantitative understanding of the quark-gluon fusion production of $h_{125}$, we normalize the fusion cross section with respect to its SM value. We define the "normalized production" factor $\mu_{\mathrm{F}}$ as

$$
\mu_{\mathrm{F}} \equiv \mu_{\mathrm{F}}^{g g+q \bar{q}}=\frac{\sigma(g g \rightarrow h)+\sum_{q=d, s, b} \sigma(q \bar{q} \rightarrow h)}{\sigma(g g \rightarrow h)_{\mathrm{SM}}} .
$$

It is a function of the BSM parameters and measures the relative enhancement of production cross section in the fusion channel. The subscript "F" stands for the fusion 


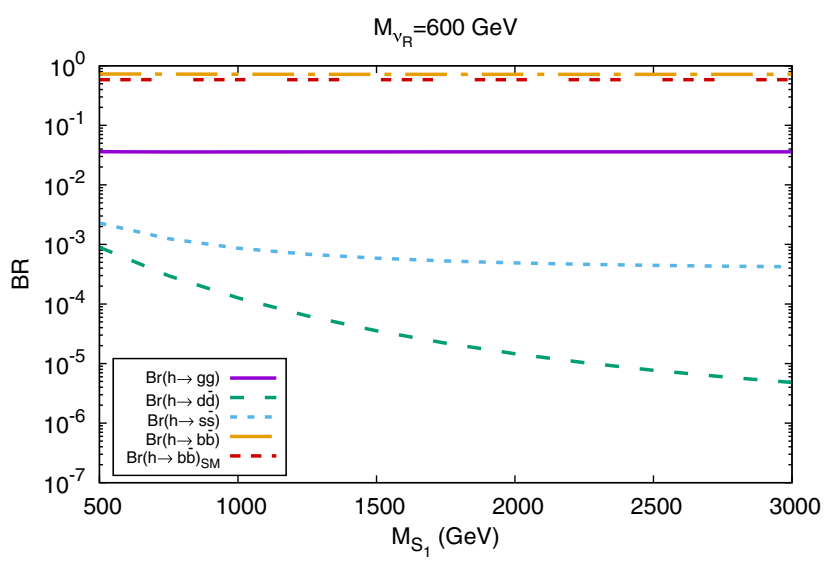

(a)

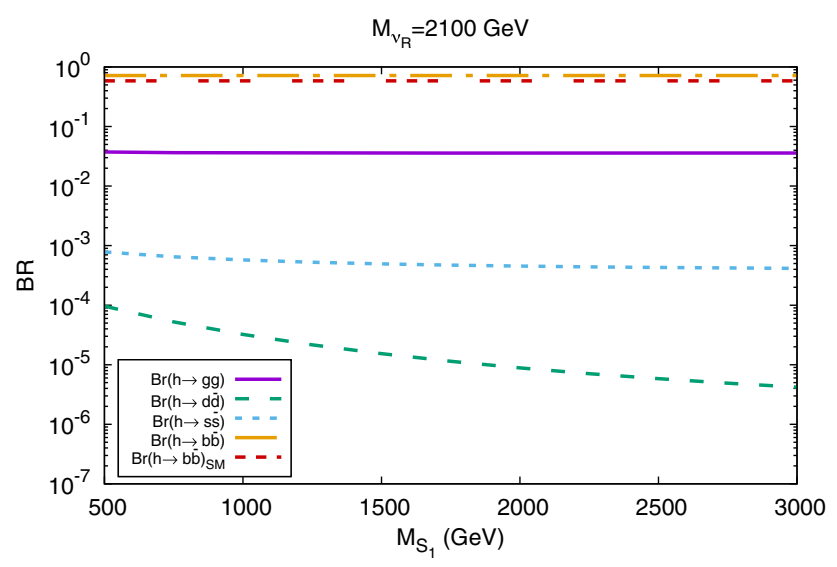

(b)

FIG. 4. Variation of $\mathrm{BR}(h \rightarrow i i)$ with $M_{S_{1}}$ for $i=d, s, b$, and gluon and two values of $M_{\nu_{R}}$ : (a) $600 \mathrm{GeV}$ and (b) $2100 \mathrm{GeV}$. We have set $g^{2} y_{\nu}=1$ for all generations and taken $\lambda=1$.

channel. In the denominator, we ignore $\sigma(b \bar{b} \rightarrow h)_{\mathrm{SM}}$, as it is much smaller than $\sigma(g g \rightarrow h)_{\mathrm{SM}}$ because of the small $b$-quark parton distribution function (PDF) in the initial states.

In our model, the leading order gluon fusion cross section at parton level can be expressed as [7,63-65]

$$
\hat{\sigma}(g g \rightarrow h)=\frac{\pi^{2} m_{h}}{8 \hat{s}} \Gamma_{h \rightarrow g g} \delta\left(\hat{s}-m_{h}^{2}\right),
$$

where $\Gamma_{h \rightarrow g g}$ is given in Eq. (23). Similarly, the quark fusion cross section at parton level can be expressed in terms of $\Gamma_{h \rightarrow q \bar{q}}$ from Eq. (22) as [62]

$$
\hat{\sigma}(q \bar{q} \rightarrow h)=\frac{4 \pi^{2} m_{h}}{9 \hat{s}} \Gamma_{h \rightarrow q \bar{q}} \delta\left(\hat{s}-m_{h}^{2}\right) .
$$

Naively, one would expect $\hat{\sigma}(q \bar{q} \rightarrow h)$ for the heavier quarks to be larger than the lighter ones, as $\Gamma_{h \rightarrow q \bar{q}}$ is proportional to the square of $y_{q}^{\text {eff }}$ (which increases linearly with $m_{q}$ ). However, there is a trade-off between $m_{q}$ and the

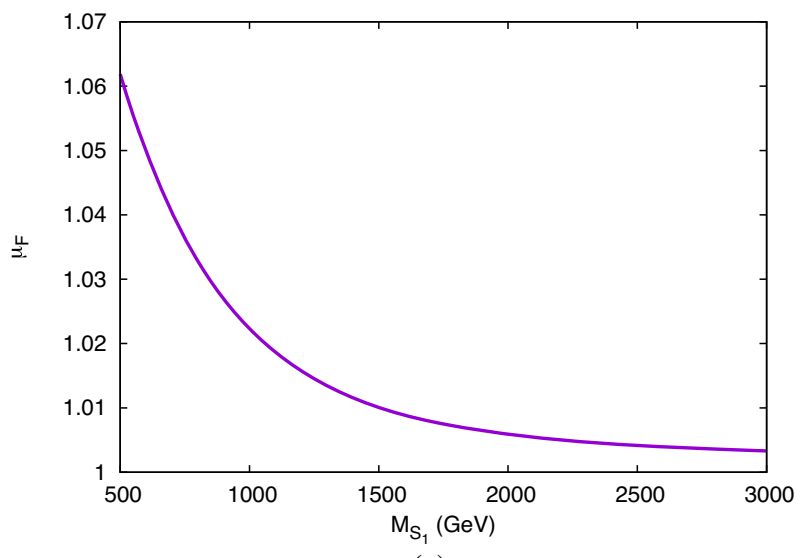

(a)

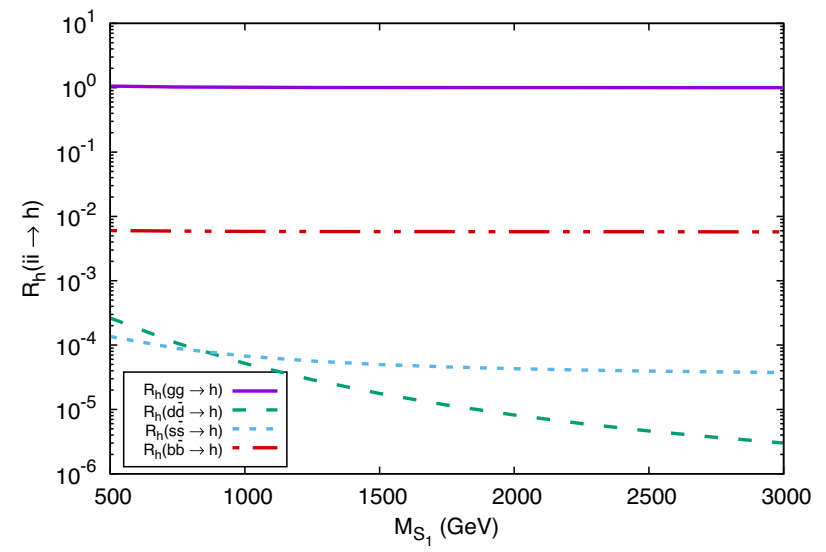

(b)

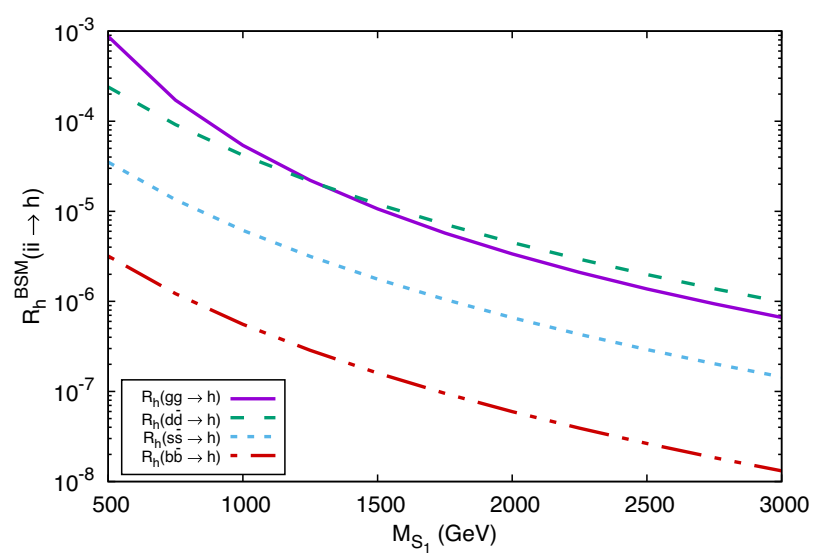

(c)

FIG. 5. (a) The normalized production cross section of $h_{125}$ as a function of $M_{S_{1}}$ for $M_{\nu_{R}}=1.1 \mathrm{TeV}$. Here also, we take $g^{2} y_{\nu}=1$ for all the generations and $\lambda=1$. (b) Relative production factor $\left(\mathrm{R}_{\mathrm{h}}\right)$ (defined in the text) for the $\mathrm{SM}+\mathrm{LQ}$ scenario as a function of $M_{S_{1}}$ for $M_{\nu_{R}}=1.1 \mathrm{TeV}$. (c) Relative production factor $\mathrm{R}_{\mathrm{h}}^{\mathrm{BSM}}$ as a function of $M_{S_{1}}$ for $M_{\nu_{R}}=1.1 \mathrm{TeV}$ when the $h q \bar{q}$ (hgg) coupling at leading order in the SM is assumed to be zero.

PDFs, as the heavier quarks PDFs are suppressed compared to their lighter counterparts. We compute $\sigma(q \bar{q} \rightarrow h)$ at the $14 \mathrm{TeV}$ LHC using the NNPDF2.3QED LO [66] PDF. Similarly, we use the next-to-next-to-leading-order plus 
next-to-next-to-leading-logarithmic QCD prediction for the $14 \mathrm{TeV}$ LHC which leads to $\sigma(g g \rightarrow h)_{\mathrm{SM}} \simeq 49.47 \mathrm{pb}$ [67]. We use these results to compute $\mu_{\mathrm{F}}$. We show $\mu_{\mathrm{F}}$ as a function of $M_{S_{1}}$ in Fig. 5(a) while assuming that $g^{2} y_{\nu}=1$ for all the generations and $\lambda=1$. For this plot, we set $M_{\nu_{R}}=1 \mathrm{TeV}$. However, since the gluon fusion cross section is much larger than the quark fusion ones, $\mu_{\mathrm{F}}$ is largely insensitive to $M_{\nu_{R}}$.

To get an idea of the contributions of the different modes to $\mu_{\mathrm{F}}$, we define the following two ratios:

$$
\begin{array}{r}
R_{h}(i i \rightarrow h)=\frac{\sigma(i i \rightarrow h)}{\sigma(g g \rightarrow h)_{\mathrm{SM}}} \quad \text { (full model), } \\
R_{h}^{\mathrm{BSM}}(i i \rightarrow h)=\frac{\sigma(i i \rightarrow h)_{\mathrm{BSM}}}{\sigma(g g \rightarrow h)_{\mathrm{SM}}} \quad \text { (BSM only). }
\end{array}
$$

The difference between these two ratios lies in the interference between the SM and BSM contributions. We show these ratios in Figs. 5(b) and 5(c). We find that, even after the PDF suppression, $R_{h}(b \bar{b} \rightarrow h)>R_{h}(s \bar{s} \rightarrow h)>$ $R_{h}(d \bar{d} \rightarrow h)$. On the other hand, if we take $R_{h}^{\mathrm{BSM}}$, the hierarchy is reversed. This can be understood from the fact that the loop contribution $\delta y$ is equal for all three of the quarks and hence the PDF suppression makes $R_{h}^{\mathrm{BSM}}(b \bar{b} \rightarrow h)<R_{h}^{\mathrm{BSM}}(s \bar{s} \rightarrow h)<R_{h}^{\mathrm{BSM}}(d \bar{d} \rightarrow h)$. Of course, because of the large gluon PDF, $\sigma(g g \rightarrow h)$ is larger than any quark fusion cross section.

\section{LIMITS ON PARAMETERS}

Any increase in either the productions or the decays of $h_{125}$ would be constrained by the existing measurements [3] (also see [68] for future projections). However, we see from Figs. 4 and 5 that the parameters we consider, i.e., $g_{i}^{2}=y_{i}^{L L} y_{i}^{\overline{R R}}=1, y_{i}^{R R}=0, \lambda=1, y_{\nu}=1$, and $\mathrm{TeV}$ scale $M_{S_{1}}, M_{\nu_{R}}$ for all three generations are quite consistent with the present and future $h_{125}$ limits.

Concerning the bounds on $S_{1}$, we see that in our parameter region of interest, LQ $S_{1}$ can decay to all the SM fermions. According to Eqs. (4) and (7), a heavy $S_{1}$ would have six decay modes for $M_{S_{1}} \leq M_{\nu_{R}}$,

$$
S_{1} \rightarrow\{u e, c \mu, t \tau, d \nu, s \nu, b \nu\},
$$

with roughly equal BR $(\sim 1 / 6)$ in each mode (if we ignore the differences among the masses of the decay products in different modes). The LHC has put exclusion bounds on scalar leptoquarks in the light-leptons + jets $(\ell \ell j j / \ell \nu j j)$ [69-71] and $b b \nu \nu / t t \tau \tau$ [72-74] channels (see also [75,76]). The strongest exclusion limit $(\sim 1.5 \mathrm{TeV})$ comes from the $\ell \ell j j$ channel for $100 \% \mathrm{BR}$ in the $S_{1} \rightarrow \ell j$ decay. These searches are for pair production of scalar leptoquarks, where the observable signal cross sections are proportional to the square of the BR involved. Hence, in our case, the limit on $S_{1}$ would get much weaker. A conservative estimation indicates that the limit goes below a $\mathrm{TeV}$ when the BR decreases to about $1 / 6$. Also, pair productions of leptoquarks are QCD driven and thus cannot be used to put limits on the fermion couplings. The CMS Collaboration has performed a search with the $8 \mathrm{TeV}$ data for single production of scalar leptoquarks that excludes up to $1.75 \mathrm{TeV}$ for order 1 coupling to the first generation [77]. However, even that limit comes down below $1 \mathrm{TeV}$ once we account for the reduction in the BR. However, a recast of CMS $8 \mathrm{TeV}$ data for the first generation $(e e j j / e \nu j j)$ indicates that for order $1 g_{(L / R)}, M_{S_{1}} \gtrsim$ 1.1 TeV [78]. ${ }^{3}$ To be on the conservative side, we may use $M_{S_{1}} \gtrsim 1.5 \mathrm{TeV}$ as a mass limit for $S_{1}$ with $g^{2} y_{\nu}=1$ for all generations.

If, however, $M_{S_{1}}>M_{\nu_{R}}$, the LQ can decay to three more final states with right-handed neutrinos. Thus, we would expect a further reduction of the limits on $S_{1}$ [27]. Moreover, specifically for first generation fermions, the choices of $g_{L}$ and $g_{R}$ are restricted further. The atomic parity violation measurements in $\mathrm{Cs}^{133}$ [79] put a strong constraint on them. Typically, all existing constraints may be satisfied easily for $M_{S_{1}} \gtrsim 2 \mathrm{TeV}$ and $g^{2} \approx 1$ with $g_{L}=g_{R}$.

\section{THE SINGLET HIGGS $\phi$}

Unlike the case of $h_{125}$, the parameters of the singlet scalar defined in Eq. (4) are largely unconstrained. Generally, to probe a heavy BSM scalar, its decays to fermion pairs like $\tau \tau$ or the massive gauge bosons are assumed to be promising. But, for a singlet scalar, these decay modes lose importance. Also, most of the BSM singlet scalar searches rely on the mixing among the singlet state with the doublet one(s), either $h_{125}$ or other BSM heavy Higgs states. In our model, by contrast, $\phi$ 's can be produced from and decay to a pair of gluons or quarks via the loop of $S_{1}$ and neutrinos without relying, in general, on the mixing of $\phi$ with the doublet Higgs. Hence, its phenomenology at the hadron collider would be different than what is generally considered in the literature.

\section{A. Effective coupling}

We first calculate the effective couplings of $\phi$ to the light quarks, as we did for $h_{125}$. The $\phi q \bar{q}$ effective coupling $Y_{q}^{\text {eff }}$ (where $q$ is any down-type quark) would receive contribution from diagrams like the one shown in Fig. 6, which is similar to the one shown in Fig. 1(b). Because of the singlet

\footnotetext{
${ }^{3}$ Recasting limits from the single production searches is trickier than the pair production case because here the production processes also depend on the unknown couplings. Even though the parton-level cross section scales easily with these couplings, one cannot account for the PDF variation for different quarks in such a simple manner. Since we are interested in a conservative limit, we have ignored the PDF variation to obtain this number.
} 


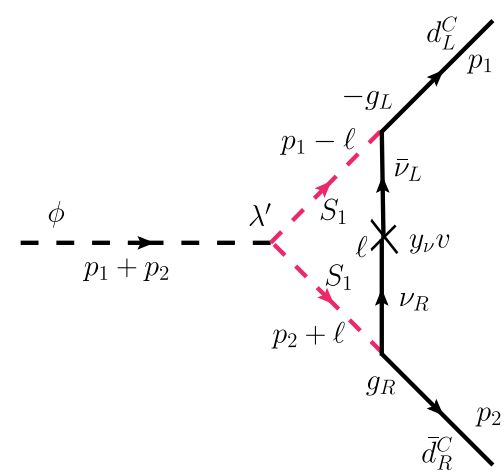

FIG. 6. The singlet scalar, $\phi$ decaying to down-type quarks.

nature of $\phi$, the tree-level $\phi \bar{\nu}_{L} \nu_{R}$ coupling does not exist, so in this case there is no diagram like the one shown in Fig. 1 (a). Proceeding as before, we get

$$
Y_{q}^{\mathrm{eff}}=\frac{g^{2} \lambda^{\prime} y_{\nu} v}{16 \pi^{2}} \int_{0}^{1} d x \int_{0}^{1-x} d y\left(\frac{1}{D_{\phi}}\right)
$$

where

$$
D_{\phi}(x, y)=M_{\nu_{R}}^{2}+(x+y)\left(M_{S_{1}}^{2}-M_{\nu_{R}}^{2}\right)-x y M_{\phi}^{2} .
$$

Written in terms of PV integrals, this becomes

$$
Y_{q}^{\mathrm{eff}}=-\frac{g^{2} \lambda^{\prime} y_{\nu} v}{16 \pi^{2}} C_{0}\left(0,0, M_{\phi}^{2}, M_{S_{1}}^{2}, M_{\nu_{R}}^{2}, M_{S_{1}}^{2}\right) .
$$

We present our results in Fig. 7, which shows the variation of $Y_{q}^{\text {eff }}$ as a function of $M_{S_{1}}$ for two values of $M_{\nu_{R}}$ and $M_{\phi}=500 \mathrm{GeV}$. Here, $\lambda^{\prime}$ is a dimensionful parameter [see Eq. (4)] that can be taken to be of the order of the largest mass in the model spectrum. The coupling $Y_{q}^{\text {eff }}$ decreases as $M_{S_{1}}$ increases. Since $\phi$ has only

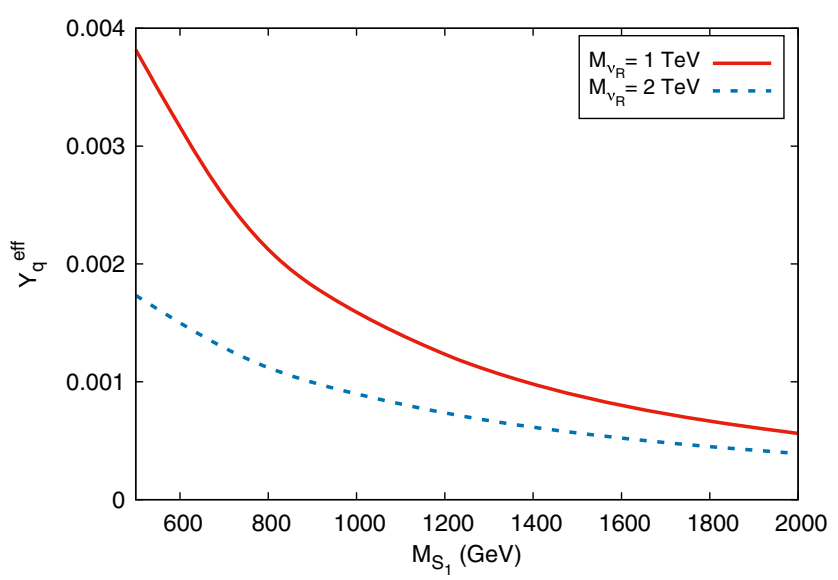

FIG. 7. Variation of $\phi q \bar{q}$ coupling as a function of $M_{S_{1}}$ for $M_{\nu_{R}}=1 \mathrm{TeV} \quad$ and $2 \mathrm{TeV}, \quad g^{2} y_{\nu}=1, \quad \lambda^{\prime}=2 \mathrm{TeV}, \quad$ and $M_{\phi}=500 \mathrm{GeV}$. loop-level interaction with the SM quarks, the effective coupling is the same for all three generations of down-type quarks for the same value of $g^{2} y_{\nu}$.

\section{B. Branching ratios and cross sections}

The expressions for the partial decay widths and production cross section of $\phi$ are essentially identical to the ones for $h_{125}$ if we replace $y_{q}^{\text {eff }} \rightarrow Y_{q}^{\text {eff }}$ and $m_{h} \rightarrow M_{\phi}$. Thus, the expressions for the partial decay widths would look like

$$
\begin{gathered}
\Gamma_{\phi \rightarrow q \bar{q}}=\frac{3\left|Y_{q}^{\mathrm{eff}}\right|^{2}}{8 \pi M_{\phi}^{2}}\left(M_{\phi}^{2}-4 m_{q}^{2}\right)^{3 / 2} \approx \frac{3}{8 \pi}\left|Y_{q}^{\mathrm{eff}}\right|^{2} M_{\phi}, \\
\Gamma_{\phi \rightarrow g g}=\frac{G_{\mathrm{F}} \alpha_{S}^{2} M_{\phi}^{3}}{64 \sqrt{2} \pi^{3}}\left|\frac{\lambda^{\prime} v}{2 M_{S_{1}}^{2}} \mathcal{A}_{0}\left(\frac{M_{\phi}^{2}}{4 M_{S_{1}}^{2}}\right)\right|^{2}, \\
\Gamma_{\phi \rightarrow \gamma \gamma}=\frac{G_{\mathrm{F}} \alpha_{\mathrm{em}}^{2} M_{\phi}^{3}}{128 \sqrt{2} \pi^{3}}\left|\frac{\lambda^{\prime} v}{6 M_{S_{1}}^{2}} \mathcal{A}_{0}\left(\frac{M_{\phi}^{2}}{4 M_{S_{1}}^{2}}\right)\right|^{2} .
\end{gathered}
$$

The Feynman diagrams for the $\phi \rightarrow \gamma \gamma$ process will be similar to those in Figs. 1(d) and 1(e), with the gluons replaced by two photons and the $\alpha_{s}$ coupling substituted for the $\alpha_{\mathrm{em}}$ coupling. As earlier, we can now express the cross sections in these modes in terms of the partial widths. In the gg channel,

$$
\hat{\sigma}(g g \rightarrow \phi)=\frac{\pi^{2} M_{\phi}}{8 \hat{s}} \Gamma_{h \rightarrow g g} \delta\left(\hat{s}-M_{\phi}^{2}\right),
$$

and in the $q \bar{q}$ channel

$$
\hat{\sigma}(q \bar{q} \rightarrow \phi)=\frac{4 \pi^{2} M_{\phi}}{9 \hat{s}} \Gamma_{\phi \rightarrow q \bar{q}} \delta\left(\hat{s}-M_{\phi}^{2}\right) .
$$

The total width for $\phi$ can be expressed as

$$
\Gamma_{\phi}=\left(\sum_{q=d, s, b} \Gamma_{\phi \rightarrow q \bar{q}}+\Gamma_{\phi \rightarrow g g}+\Gamma_{\phi \rightarrow \gamma \gamma}\right) .
$$

We now present our numerical results. We begin with Fig. 8, where we show the variation of BRs of different decay modes of $\phi$. For the most part, the plots for the quarks overlap, as $\Gamma_{\phi \rightarrow q \bar{q}}$ is essentially independent of $\mathrm{m}_{q}$ [see Eq. (37)]. Here, without any singlet-doublet mixing, $\phi$ can decay only to down-type quarks or gluon or photon pairs. As a result, when $M_{S_{1}}$ increases, $\operatorname{BR}(\phi \rightarrow g g / \gamma \gamma)$ decreases and $\operatorname{BR}(\phi \rightarrow q \bar{q})$ goes up if $M_{\nu_{R}}$ is held fixed. We see that for a $2 \mathrm{TeV} S_{1}, \phi \rightarrow q \bar{q}$ is the dominant decay mode for $g^{2} y_{\nu}=1, M_{\nu_{R}}=1 \mathrm{TeV}$ (the BRs are independent of $\left.\lambda^{\prime}\right)$.

In Figs. 9(a) and 9(b), we plot the scattering cross sections of $\phi$ in different decay modes at the $14 \mathrm{TeV}$ LHC, considering both the gluon and quark fusion processes. We 


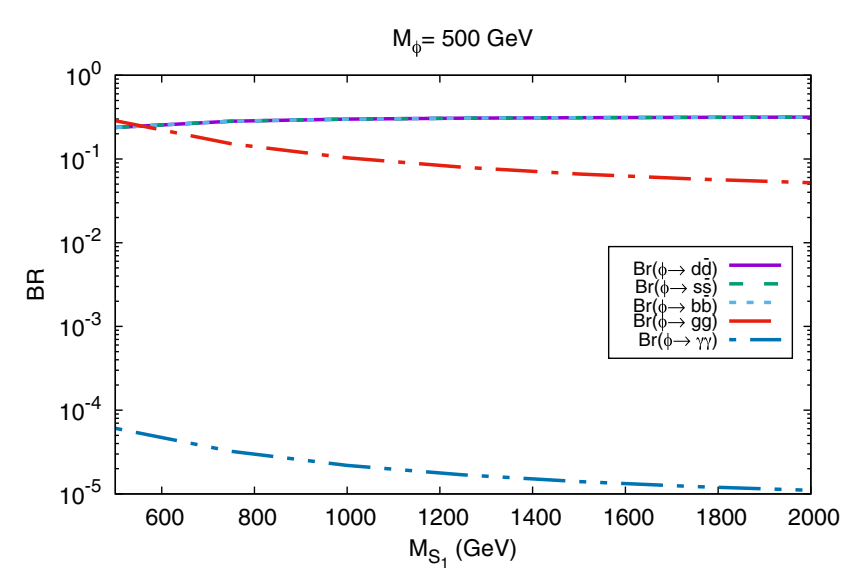

(a)

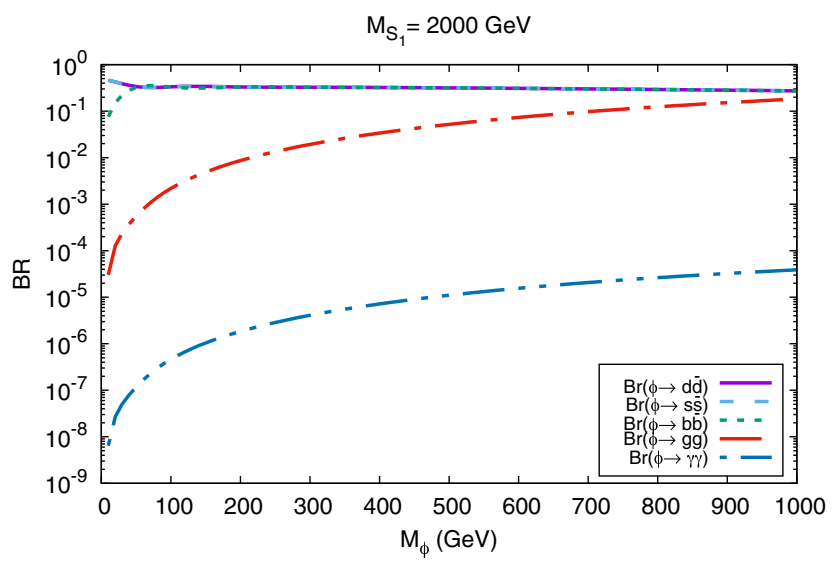

(b)

FIG. 8. Variation of BR $(\phi \rightarrow q \bar{q})$, BR $(\phi \rightarrow g g)$, and BR $(\phi \rightarrow$ $\gamma \gamma$ ) as a function of (a) $M_{S_{1}}$ and (b) $M_{\phi}$ for $g^{2} y_{\nu}=1$ and $M_{\nu_{R}}=1 \mathrm{TeV}$. The ratios are independent of $\lambda^{\prime}$. We set $\lambda^{\prime}=$ $2 \mathrm{TeV}$ to compute the partial decay widths of $\phi$.

show the production cross section times the branching ratio for all the modes against $M_{S_{1}}$ and $M_{\phi}$. Note that, in the parameter space that we consider, we find $\Gamma_{\phi} \ll M_{\phi}$, which makes the narrow width approximation used in our computation a valid one. Here, we use the same set of PDFs as in the $h_{125}$ case. To have some intuition about the strengths of different production channels, we scale the cross sections by $\sigma\left(g g \rightarrow h_{M_{\phi}}\right)$, where $h_{M_{\phi}}$ represents a BSM Higgs whose couplings with the SM particles are the same as those of $h_{125}$. Its production cross section in the gluon fusion mode can be computed from Eq. (30), after taking $M_{S_{1}} \rightarrow \infty$ in Eq. (23), as

$\hat{\sigma}\left(g g \rightarrow h_{M_{\phi}}\right) \simeq \frac{G_{\mathrm{F}} \alpha_{S}^{2} M_{\phi}^{4}}{512 \sqrt{2} \pi \hat{s}}\left|\mathcal{A}_{1 / 2}\left(\frac{M_{\phi}^{2}}{4 m_{t}^{2}}\right)\right|^{2} \delta\left(\hat{s}-M_{\phi}^{2}\right)$.

Then we define the scaled cross sections as

$$
R_{\phi}(i i \rightarrow \phi)=\frac{\sigma(i i \rightarrow \phi)}{\sigma\left(g g \rightarrow h_{M_{\phi}}\right)} .
$$

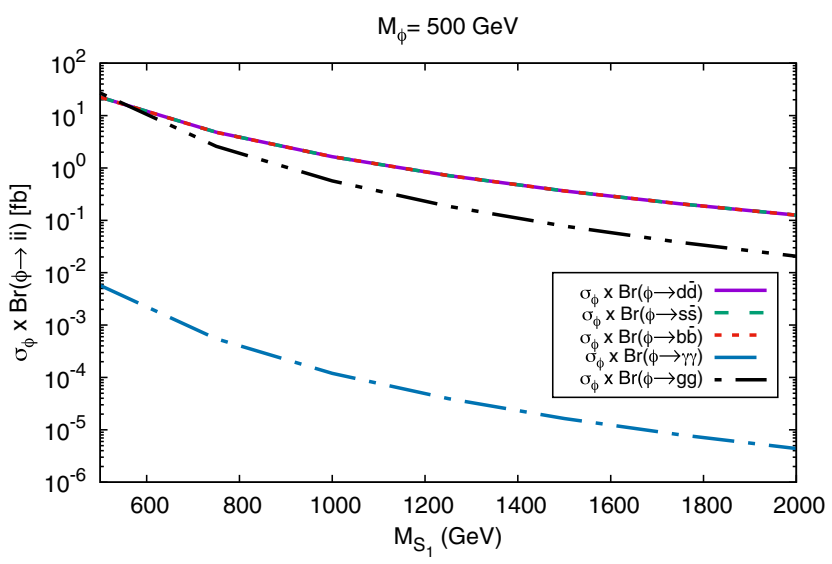

(a)

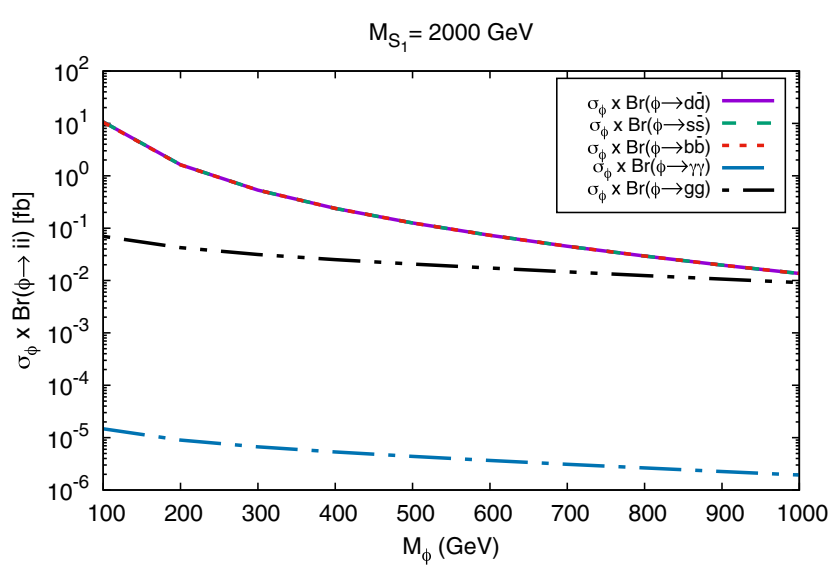

(b)

FIG. 9. Variation of the production cross section of $\phi$ times the branching ratios as functions of (a) $M_{S_{1}}$ and (b) $M_{\phi}$ for $g^{2} y_{\nu}=1$, $\lambda^{\prime}=2 \mathrm{TeV}$, and $M_{\nu_{R}}=1 \mathrm{TeV}$ at the $14 \mathrm{TeV}$ LHC.

In Figs. 10(a) and 10(b), we show the variation of $R_{\phi}$ with $M_{S_{1}}$ and $M_{\phi}$. Recall that a SM singlet $\phi$ cannot be produced at tree level. The leading order contribution to $\sigma(i i \rightarrow \phi)$ starts at one-loop level. In Fig. 10(b), we observe a crossover where the qqF becomes the dominant process over the ggF, i.e., $\sigma(q \bar{q} \rightarrow \phi)>\sigma(g g \rightarrow \phi)$ for a fixed value of LQ mass $(=2 \mathrm{TeV})$. This is not a generic pattern and can be understood from Eqs. (37) and (38) by varying a few of the free parameters. For example, for a relatively large value of LQ mass $\left(M_{S_{1}} \geq 2 \mathrm{TeV}\right)$, one may obtain $\Gamma_{\phi \rightarrow g g} \leq \Gamma_{\phi \rightarrow q \bar{q}}$ when $\phi$ is not large, i.e., $M_{\phi} \leq 250 \mathrm{GeV}$. In this case, the quark fusion process would have leading contributions. If one increases $M_{S_{1}}$ further, $\Gamma_{\phi \rightarrow g g}$ decreases more rapidly than $\Gamma_{\phi \rightarrow q \bar{q}}$, with $M_{\phi}$ ensuring that the $q \bar{q} \rightarrow \phi$ process remains the dominant one for a larger range of $M_{\phi}$. For example, if one sets $M_{S_{1}} \sim 3 \mathrm{TeV}$, we find that quark fusion becomes dominant for $M_{\phi} \leq 350 \mathrm{GeV}$. However, the relative contributions are insensitive to the value of $\lambda^{\prime}$ chosen. 


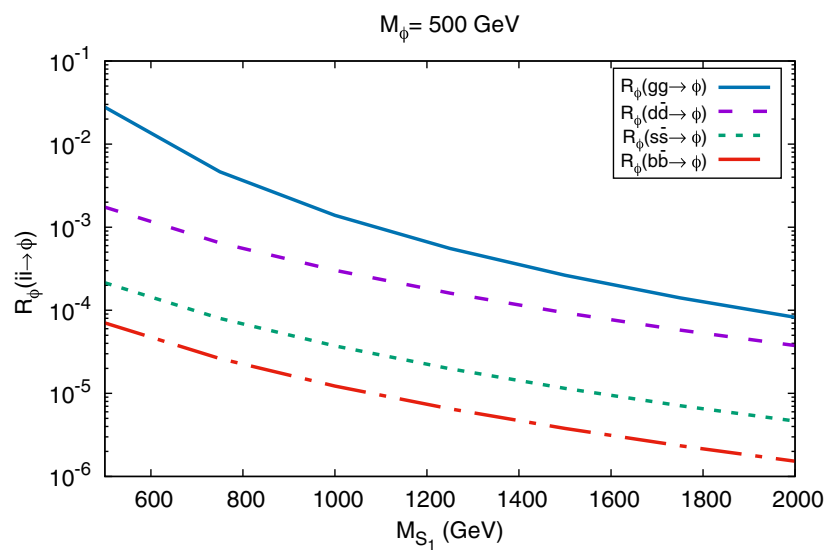

(a)

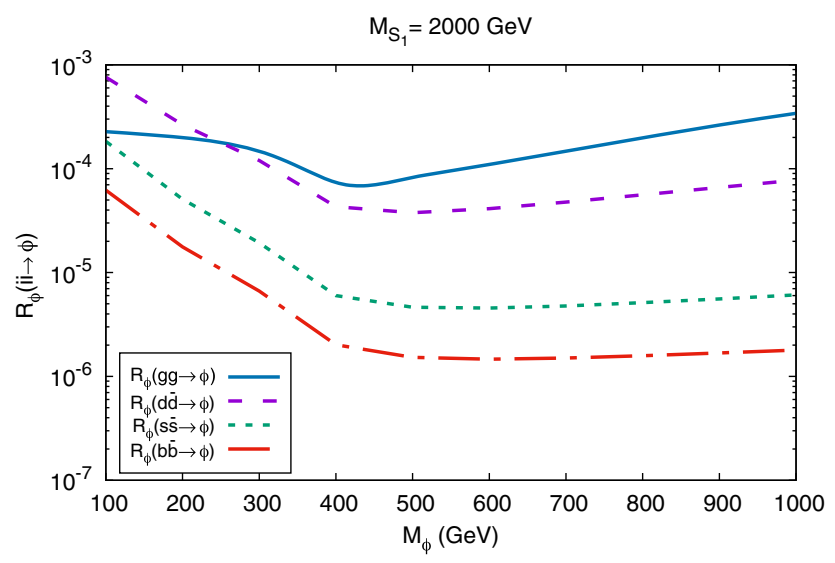

(b)

FIG. 10. Variation of $R_{\phi}(i i \rightarrow \phi)$ [Eq. (44)] with (a) $M_{S_{1}}$ and (b) $M_{\phi}$ for $g^{2} y_{\nu}=1, \lambda^{\prime}=2 \mathrm{TeV}$, and $M_{\nu_{R}}=1 \mathrm{TeV}$.

\section{Prospects at the LHC}

It is clear that the scalar $\phi$ in our model would offer some novel and interesting phenomenology at the LHC. However, a detailed analysis is beyond the scope of this paper. Instead we now simply make a few comments on its prospects.

It may be possible to put a bound on $\sigma_{\phi}\left(M_{\phi}\right)$ from the dijet resonance searches. For example, the one performed by the CMS Collaboration at the $13 \mathrm{TeV}$ LHC [80] indicates that $\sigma_{\phi} \times \mathrm{BR}(\phi \rightarrow g g)$ has to be less than about $1 \mathrm{pb}$ for $M_{\phi}=1 \mathrm{TeV}$ and about $20 \mathrm{pb}$ for $M_{\phi}=600 \mathrm{GeV}$. Similarly, in the quark mode, $\sigma_{\phi} \times \mathrm{BR}(\phi \rightarrow d \bar{d}+s \bar{s}+b \bar{b})$ is less than about $1 \mathrm{pb}$ for $M_{\phi}=1 \mathrm{TeV}$ and about $5 \mathrm{pb}$ for $M_{\phi}=600 \mathrm{GeV}$. Figure 9(b) (which is obtained for the $14 \mathrm{TeV}$ LHC) indicates that our choice of parameters easily satisfies this limit. Future searches in this channel would put stronger bounds on $\sigma_{\phi}$ and/or $M_{\phi}$. The LHC has also searched for such a state in the $\gamma \gamma$ final states, though the present bound from this channel is weaker [81] than the dijet one. In our model, this channel is not at all promising, as can be seen in Figs. 9 and 10. Even the HL-LHC might not be able to probe the singlet state in the $\gamma \gamma$ mode.

\section{CONCLUSION}

In this paper, we have considered a simple extension to the SM, in which we have a scalar LQ $\left(S_{1}\right)$ with electromagnetic charge $1 / 3$ and heavy right chiral neutrinos. While the presence of both BSM particles may have its origin in a grand unified framework, we have simply considered their interactions at the $\mathrm{TeV}$ scale. The motivation for considering such an extension comes from the fact that it can accommodate Yukawa couplings of the down-type quarks that are enhanced compared to SM expectations.

We have shown that the LQ and the right chiral neutrinos can enhance the production cross section of the SM-like Higgs through a triangle loop. We have calculated the oneloop contributions to the Yukawa couplings of the downtype quarks. We have found the enhancements (which we have parametrized by the usual $\kappa_{d, s, b}$ ) for order 1 new couplings and $\mathrm{TeV}$ scale new particles. We have then further extended our analysis to include a SM-singlet scalar $\phi$ in the model with a dimension-1 coupling with $S_{1}$ but no tree-level mixing with the SM-like Higgs. We have found that, for a similar choice of parameters, the gluon fusion (through a LQ in the loop) and the quark fusion (mediated by a LQ and neutrinos in a loop) processes can lead to a significant cross section to produce $\phi$ at the LHC. They also enhance the decay width of the singlet. Interestingly, we have found that for a light $\phi$, the quark fusion can become more important than the gluon fusion process as long as the mass of the LQ remains high $(\sim \mathrm{TeV})$. In both cases, precise measurements of branching fractions or partial widths of the $125 \mathrm{GeV}$ SM-like Higgs or the singlet scalar, i.e., $h_{125}$, $\phi \rightarrow d \bar{d}, s \bar{s}, b \bar{b}$, would be crucial for testing or constraining the model at the high luminosity run of the LHC.

\section{ACKNOWLEDGMENTS}

Our computations were supported in part by SAMKHYA: the high performance computing facility provided by the Institute of Physics (IoP), Bhubaneswar, India. A. B. and S. M. acknowledge support from the Science and Engineering Research Board (SERB), DST, India, under Grant No. ECR/2017/000517. We thank P. Agrawal for the helpful discussion. S. M. also acknowledges the local hospitality at IoP, Bhubaneswar during the meeting IMHEP-19, where this work was initiated. 
[1] G. Aad et al. (ATLAS Collaboration), Observation of a new particle in the search for the Standard Model Higgs boson with the ATLAS detector at the LHC, Phys. Lett. B 716, 1 (2012).

[2] S. Chatrchyan et al. (CMS Collaboration), Observation of a new boson at a mass of $125 \mathrm{GeV}$ with the CMS Experiment at the LHC, Phys. Lett. B 716, 30 (2012).

[3] G. Aad et al. (ATLAS Collaboration), Combined measurements of Higgs boson production and decay using up to $80 \mathrm{fb}^{-1}$ of proton-proton collision data at $\sqrt{s}=13 \mathrm{TeV}$ collected with the ATLAS experiment, Phys. Rev. D 101, 012002 (2020).

[4] J. C. Pati and A. Salam, Unified lepton-hadron symmetry and a gauge theory of the basic interactions, Phys. Rev. D 8, 1240 (1973).

[5] H. Georgi and S. L. Glashow, Unity of All ElementaryParticle Forces, Phys. Rev. Lett. 32, 438 (1974).

[6] H. Georgi, The state of the art-Gauge theories, AIP Conf. Proc. 23, 575 (1975).

[7] I. Doršner, S. Fajfer, A. Greljo, J. F. Kamenik, and N. Košnik, Physics of leptoquarks in precision experiments and at particle colliders, Phys. Rep. 641, 1 (2016).

[8] J. Blumlein, E. Boos, and A. Kryukov, Leptoquark pair production in hadronic interactions, Z. Phys. C 76, 137 (1997).

[9] S. Fajfer and N. Košnik, Vector leptoquark resolution of $R_{K}$ and $R_{D^{(*)}}$ puzzles, Phys. Lett. B 755, 270 (2016).

[10] R. Barbieri, G. Isidori, A. Pattori, and F. Senia, Anomalies in $B$-decays and $U(2)$ flavour symmetry, Eur. Phys. J. C 76, 67 (2016).

[11] I. Doršner, S. Fajfer, N. Košnik, and I. Nišandžić, Minimally flavored colored scalar in $\bar{B} \rightarrow D^{(*)} \tau \bar{\nu}$ and the mass matrices constraints, J. High Energy Phys. 11 (2013) 084.

[12] B. Gripaios, M. Nardecchia, and S. A. Renner, Composite leptoquarks and anomalies in $B$-meson decays, J. High Energy Phys. 05 (2015) 006.

[13] D. Bečirević, S. Fajfer, and N. Košnik, Lepton flavor nonuniversality in $b \rightarrow s \ell^{+} \ell^{-}$processes, Phys. Rev. D 92, 014016 (2015).

[14] D. Bečirević, S. Fajfer, N. Košnik, and O. Sumensari, Leptoquark model to explain the $B$-physics anomalies, $R_{K}$ and $R_{D}$, Phys. Rev. D 94, 115021 (2016).

[15] A. Crivellin, D. Müller, and T. Ota, Simultaneous explanation of $R\left(D^{(*)}\right)$ and $b \rightarrow s \mu^{+} \mu^{-}$: The last scalar leptoquarks standing, J. High Energy Phys. 09 (2017) 040.

[16] J. M. Cline, $B$ decay anomalies and dark matter from vectorlike confinement, Phys. Rev. D 97, 015013 (2018).

[17] L. Di Luzio and M. Nardecchia, What is the scale of new physics behind the $B$-flavour anomalies?, Eur. Phys. J. C 77, 536 (2017).

[18] T. Mandal, S. Mitra, and S. Raz, $R_{D^{(*)}}$ motivated $\mathcal{S}_{1}$ leptoquark scenarios: Impact of interference on the exclusion limits from LHC data, Phys. Rev. D 99, 055028 (2019).

[19] U. Aydemir, T. Mandal, and S. Mitra, Addressing the $\mathbf{R}_{D^{(*)}}$ anomalies with an $\mathbf{S}_{1}$ leptoquark from $\mathbf{S O}(\mathbf{1 0})$ grand unification, Phys. Rev. D 101, 015011 (2020).

[20] A. Crivellin, D. Müller, and F. Saturnino, Flavor phenomenology of the leptoquark singlet-triplet model, J. High Energy Phys. 06 (2020) 020.
[21] K. Cheung, W. Y. Keung, and P. Y. Tseng, Leptoquark induced rare decay amplitudes $h \rightarrow \tau^{\mp} \mu^{ \pm}$and $\tau \rightarrow \mu \gamma$, Phys. Rev. D 93, 015010 (2016).

[22] R. Mandal, Fermionic dark matter in leptoquark portal, Eur. Phys. J. C 78, 726 (2018).

[23] S.-M. Choi, Y.-J. Kang, H. M. Lee, and T.-G. Ro, Leptoquark portal dark matter, J. High Energy Phys. 10 (2018) 104.

[24] A. Djouadi, T. Köhler, M. Spira, and J. Tutas, (eb), (et) type leptoquarks at ep colliders, Z. Phys. C 46, 679 (1990).

[25] K.-m. Cheung, Muon anomalous magnetic moment and leptoquark solutions, Phys. Rev. D 64, 033001 (2001).

[26] I. Doršner, S. Fajfer, and O. Sumensari, Muon $g-2$ and scalar leptoquark mixing, J. High Energy Phys. 06 (2020) 089.

[27] D. Das, K. Ghosh, M. Mitra, and S. Mondal, Probing sterile neutrinos in the framework of inverse seesaw mechanism through leptoquark productions, Phys. Rev. D 97, 015024 (2018).

[28] P. Bandyopadhyay and R. Mandal, Vacuum stability in an extended Standard Model with a leptoquark, Phys. Rev. D 95, 035007 (2017).

[29] T. Mandal, S. Mitra, and S. Seth, Pair production of scalar leptoquarks at the LHC to NLO parton shower accuracy, Phys. Rev. D 93, 035018 (2016).

[30] I. Doršner, S. Fajfer, D. A. Faroughy, and N. Košnik, The role of the $S_{3}$ GUT leptoquark in flavor universality and collider searches, J. High Energy Phys. 10 (2017) 188.

[31] P. Bandyopadhyay and R. Mandal, Revisiting scalar leptoquark at the LHC, Eur. Phys. J. C 78, 491 (2018).

[32] G. Hiller, D. Loose, and I. Nišandžić, Flavorful leptoquarks at hadron colliders, Phys. Rev. D 97, 075004 (2018).

[33] A. Biswas, A. Shaw, and A. K. Swain, Collider signature of $V_{2}$ leptoquark with $b \rightarrow s$ flavour observables, Lett. High Energy Phys. 2, 126 (2019).

[34] T. Faber, Y. Liu, W. Porod, M. Hudec, M. Malinský, F. Staub, and H. Kolešová, Collider phenomenology of a unified leptoquark model, Phys. Rev. D 101, 095024 (2020).

[35] A. Alves, O. J. P. Eboli, G. G. Di Cortona, and R. R. Moreira, Indirect and monojet constraints on scalar leptoquarks, Phys. Rev. D 99, 095005 (2019).

[36] K. Chandak, T. Mandal, and S. Mitra, Hunting for scalar leptoquarks with boosted tops and light leptons, Phys. Rev. D 100, 075019 (2019).

[37] R. Padhan, S. Mandal, M. Mitra, and N. Sinha, Signatures of $\tilde{R}_{2}$ class of leptoquarks at the upcoming ep colliders, Phys. Rev. D 101, 075037 (2020).

[38] B. C. Allanach, T. Corbett, and M. Madigan, Sensitivity of future hadron colliders to leptoquark pair production in the di-muon di-jets channel, Eur. Phys. J. C 80, 170 (2020).

[39] R. N. Mohapatra, Mechanism for Understanding Small Neutrino Mass in Superstring Theories, Phys. Rev. Lett. 56, 561 (1986).

[40] S. Nandi and U. Sarkar, Solution to the Neutrino-Mass Problem in Superstring $\mathbf{E}_{6}$ Theory, Phys. Rev. Lett. 56, 564 (1986).

[41] R. N. Mohapatra and J. W. F. Valle, Neutrino mass and baryon-number nonconservation in superstring models, Phys. Rev. D 34, 1642 (1986). 
[42] F. Deppisch and J. W. F. Valle, Enhanced lepton flavor violation in the supersymmetric inverse seesaw model, Phys. Rev. D 72, 036001 (2005).

[43] A. Abada, D. Das, and C. Weiland, Enhanced Higgs mediated lepton flavour violating processes in the supersymmetric inverse seesaw model, J. High Energy Phys. 03 (2012) 100.

[44] A. Abada, D. Das, A. Vicente, and C. Weiland, Enhancing lepton flavour violation in the supersymmetric inverse seesaw beyond the dipole contribution, J. High Energy Phys. 09 (2012) 015.

[45] S. Mondal, S. Biswas, P. Ghosh, and S. Roy, Exploring novel correlations in trilepton channels at the LHC for the minimal supersymmetric inverse seesaw model, J. High Energy Phys. 05 (2012) 134.

[46] P. S. B. Dev, S. Mondal, B. Mukhopadhyaya, and S. Roy, Phenomenology of light sneutrino dark matter in cMSSM/ mSUGRA with inverse seesaw, J. High Energy Phys. 09 (2012) 110.

[47] S. Banerjee, P. S. B. Dev, S. Mondal, B. Mukhopadhyaya, and S. Roy, Invisible Higgs Decay in a supersymmetric inverse seesaw model with light sneutrino dark matter, J. High Energy Phys. 10 (2013) 221.

[48] A. Abada, M. E. Krauss, W. Porod, F. Staub, A. Vicente, and C. Weiland, Lepton flavor violation in low-scale seesaw models: SUSY and non-SUSY contributions, J. High Energy Phys. 11 (2014) 048.

[49] E. Arganda, M. J. Herrero, X. Marcano, and C. Weiland, Imprints of massive inverse seesaw model neutrinos in lepton flavor violating Higgs boson decays, Phys. Rev. D 91, 015001 (2015).

[50] E. Arganda, M. J. Herrero, X. Marcano, and C. Weiland, Enhancement of the lepton flavor violating Higgs boson decay rates from SUSY loops in the inverse seesaw model, Phys. Rev. D 93, 055010 (2016).

[51] P. Agrawal and U. Mahanta, Leptoquark contribution to the Higgs boson production at the CERN LHC collider, Phys. Rev. D 61, 077701 (2000).

[52] S. Bar-Shalom and A. Soni, Universally enhanced lightquarks Yukawa couplings paradigm, Phys. Rev. D 98, 055001 (2018).

[53] L. Alasfar, R. C. Lopez, and R. Gröber, Probing Higgs couplings to light quarks via Higgs pair production, J. High Energy Phys. 11 (2019) 088.

[54] J. de Blas et al., Higgs Boson studies at future particle colliders, J. High Energy Phys. 01 (2020) 139.

[55] U. Ellwanger, C. Hugonie, and A. M. Teixeira, The next-tominimal supersymmetric standard model, Phys. Rep. 496, 1 (2010).

[56] D. Das, Dominant production of heavier Higgs bosons through vector boson fusion in the NMSSM, Phys. Rev. D 99, 095035 (2019).

[57] R. Mandal and A. Pich, Constraints on scalar leptoquarks from lepton and kaon physics, J. High Energy Phys. 12 (2019) 089.

[58] E. Arganda, M. J. Herrero, X. Marcano, R. Morales, and A. Szynkman, Effective lepton flavor violating $H \ell_{i} \ell_{j}$ vertex from right-handed neutrinos within the mass insertion approximation, Phys. Rev. D 95, 095029 (2017).
[59] G. Passarino and M. J. G. Veltman, One loop corrections for $e^{+} e^{-}$annihilation into $\mu^{+} \mu^{-}$in the Weinberg model, Nucl. Phys. B160, 151 (1979).

[60] V. Shtabovenko, R. Mertig, and F. Orellana, New developments in FeynCalc9.0, Comput. Phys. Commun. 207, 432 (2016).

[61] T. Hahn and M. Perez-Victoria, Automatized one loop calculations in four-dimensions and D-dimensions, Comput. Phys. Commun. 118, 153 (1999).

[62] M. Tanabashi et al. (Particle Data Group Collaboration), Review of particle physics, Phys. Rev. D 98, 030001 (2018).

[63] A. Djouadi, The anatomy of electro-weak symmetry breaking. II. The Higgs bosons in the minimal supersymmetric model, Phys. Rep. 459, 1 (2008).

[64] J. F. Gunion and H. E. Haber, Higgs bosons in supersymmetric models. 2. Implications for phenomenology, Nucl. Phys. B278, 449 (1986); Erratum, Nucl. Phys. B402, 569 (1993).

[65] W.-F. Chang, J. N. Ng, and J. M. S. Wu, Constraints on new scalars from the LHC $125 \mathrm{GeV}$ Higgs signal, Phys. Rev. D 86, 033003 (2012).

[66] R. D. Ball, V. Bertone, S. Carrazza, L. Del Debbio, S. Forte, A. Guffanti, N. P. Hartland, and J. Rojo, Parton distributions with QED corrections, Nucl. Phys. B877, 290 (2013).

[67] See https://twiki.cern.ch/twiki/bin/view/LHCPhysics/ CERNYellowReportPageBSMAt14TeV\#WH_Process.

[68] M. Cepeda et al., Report from Working Group 2, CERN Yellow Rep. Monogr. 7, 221 (2019).

[69] M. Aaboud et al. (ATLAS Collaboration), Searches for scalar leptoquarks and differential cross-section measurements in dilepton-dijet events in proton-proton collisions at a centre-of-mass energy of $\sqrt{s}=13 \mathrm{TeV}$ with the ATLAS experiment, Eur. Phys. J. C 79, 733 (2019).

[70] A. M. Sirunyan et al. (CMS Collaboration), Search for pair production of first-generation scalar leptoquarks at $\sqrt{s}=13$ TeV, Phys. Rev. D 99, 052002 (2019).

[71] A. M. Sirunyan et al. (CMS Collaboration), Search for pair production of second-generation leptoquarks at $\sqrt{s}=13$ TeV, Phys. Rev. D 99, 032014 (2019).

[72] A. M. Sirunyan et al. (CMS Collaboration), Constraints on models of scalar and vector leptoquarks decaying to a quark and a neutrino at $\sqrt{s}=13 \mathrm{TeV}$, Phys. Rev. D 98, 032005 (2018).

[73] A. M. Sirunyan et al. (CMS Collaboration), Search for Leptoquarks Coupled to Third-Generation Quarks in Proton-Proton Collisions at $\sqrt{s}=13 \mathrm{TeV}$, Phys. Rev. Lett. 121, 241802 (2018).

[74] M. Aaboud et al. (ATLAS Collaboration), Searches for third-generation scalar leptoquarks in $\sqrt{s}=13 \mathrm{TeV} p p$ collisions with the ATLAS detector, J. High Energy Phys. 06 (2019) 144.

[75] Y. Takahashi (CMS Collaboration), Leptoquark searches in CMS, in Proceedings of the 53rd Rencontres de Moriond on Electroweak Interactions and Unified Theories (Moriond EW 2018), La Thuile, Italy, 2018 (ARISF, 2018), pp. 65-70, http://moriond.in2p3.fr/Proceedings/2018/Moriond_EW_ 2018.pdf.

[76] V. W.S. Wong (ATLAS Collaboration), Searching for leptoquarks with the ATLAS detector, in Proceedings of the 17th Conference on Flavor Physics and CP Violation (FPCP 2019), Victoria, British Columbia, Canada, 2019 (2019), https://www.slac.stanford.edu/econf/C190506/. 
[77] V. Khachatryan et al. (CMS Collaboration), Search for single production of scalar leptoquarks in proton-proton collisions at $\sqrt{s}=8 \mathrm{TeV}$, Phys. Rev. D 93, 032005 (2016); Erratum, Phys. Rev. D 95, 039906 (2017).

[78] T. Mandal, S. Mitra, and S. Seth, Single productions of colored particles at the LHC: An example with scalar leptoquarks, J. High Energy Phys. 07 (2015) 028.

[79] P. Langacker, Parity violation in muonic atoms and cesium, Phys. Lett. B 256, 277 (1991).
[80] A. M. Sirunyan et al. (CMS Collaboration), Search for dijet resonances in proton-proton collisions at $\sqrt{s}=$ $13 \mathrm{TeV}$ and constraints on dark matter and other models, Phys. Lett. B 769, 520 (2017); Erratum, Phys. Lett. B 772, 882 (2017).

[81] M. Aaboud et al. (ATLAS Collaboration), Search for new phenomena in high-mass diphoton final states using $37 \mathrm{fb}^{-1}$ of proton-proton collisions collected at $\sqrt{s}=13 \mathrm{TeV}$ with the ATLAS detector, Phys. Lett. B 775, 105 (2017). 